\title{
BReserch S Suare \\ Grey Wolf Optimization Based Self-Organizing Fuzzy Multi-Objective Evolution Algorithm
}

\author{
Jialiang Xie ( $\nabla$ xiejialiang@jmu.edu.cn ) \\ Jimei University https://orcid.org/0000-0002-2329-2144 \\ Shanli Zhang \\ Jimei University \\ Honghui Wang \\ Jimei University \\ Dongrui Wu \\ Huazhong University of Science and Technology
}

\section{Research Article}

Keywords: Grey wolf optimization, Fuzzy inference system, Self-organizing map, Evolutionary algorithm

Posted Date: April 12th, 2021

DOl: https://doi.org/10.21203/rs.3.rs-320117/v1

License: (c) (1) This work is licensed under a Creative Commons Attribution 4.0 International License.

Read Full License 


\title{
Grey Wolf Optimization Based Self-Organizing Fuzzy Multi-Objective Evolution Algorithm
}

\author{
Jialiang Xie* · Shanli Zhang • Honghui Wang • Dongrui Wu
}

Received: date / Accepted: date

\begin{abstract}
Two goals of multi-objective evolutionary algorithms are effectively improving its convergence and diversity, and making the Pareto set evenly distributed and close to the real Pareto Front. This paper proposes a grey wolf optimization based self-organizing fuzzy multi-objective evolutionary algorithm. Grey wolf optimization algorithm is used to optimize the initial weights of the self-organizing map network. New neighborhood relationships for individuals are built by self-organizing map, which can maintain the invariance of feature distribution and map the structural information of the current population into Pareto Sets. Based on this neighborhood relationship, this paper uses the fuzzy differential evolution operator, which constructs a fuzzy inference system to dynamically adjust the weighting parameter in the differential operator, to generate a new initial solution, and the polynomial mutation operator to refine them. Boundary processing is then conducted. Experiments on fifteen test problems were conducted to verify its effectiveness. Results show that the convergence and diversity of the proposed algorithm are better than several state-of-the-art multi-objective evolutionary algorithms.
\end{abstract}

\section{Jialiang Xie}

The corresponding author. School of Science, Jimei University, Xiamen 361021, China

E-mail: xiejialiang@jmu.edu.cn

Shanli Zhang

School of Science, Jimei University, Xiamen 361021, China

E-mail: 845303782@qq.com

Honghui Wang

School of Science, Jimei University, Xiamen 361021, China

E-mail: 349638414@qq.com

Dongrui Wu

School of Artificial Intelligence and Automation, Huazhong University of Science and Technology, Wuhan 430074, China

Key Laboratory of the Ministry of Education for Image Processing and Intelligent Control, China

E-mail: drwu@ hust.edu.cn
Keywords Grey wolf optimization - Fuzzy inference system $\cdot$ Self-organizing map $\cdot$ Evolutionary algorithm

\section{Introduction}

Many optimization problems in scientific research and industrial applications are intrinsic multi-objective, in which multiple conflicting objectives need to be optimized simultaneously. Thus, it is impossible to achieve the optimality of all problems at the same time. The solution is a Pareto optimal solution set (PS), consisting of multiple compromised solutions among different objectives. Vectors in the objective space that correspond to the PS is Pareto front (PF) (Zhou et al. 2009).

Common approaches to solve multi-objective optimization problems are traditional mathematical analytical algorithms and evolutionary algorithms. Multi-objective evolutionary algorithms (MOEAs) provide a general framework for solving complex problems and have been widely used in dynamic optimization, machine learning, signal processing, adaptive control, and so on. Popular MOEAs are usually based on Pareto dominance, performance indicator and decomposition (Zhang et al. 2016).

An effective MOEA should make full use of the regularity property of multi-objective optimization problems (Zhang et al. 2016), that is, under certain conditions, the PF and PS of a continuous m-objective optimization problem form an (m-1)-dimensional piecewise continuous manifold in the objective space and the decision space, respectively. Regularity model based multi-objective estimation of distribution algorithms (Zhang et al. 2008) explicitly uses this property, for modeling the PS (Zhou et al. 2009) and for performing local search (Lara et al. 2010). Cellular multi-objective genetic algorithms (Durillo et al. 2008; Nebro et al. 2009; Zhang et al. 2015), MOEA based on decomposition (MOEAs/D) 
(Zhang et al. 2008; Li and Zhang 2009; Wang and Zhang 2016; Zhou and Zhang 2016), and hybrid NSGA-II with self-organizing map (Norouzi and Rakhshandehroo 2011) implicitly use this property.

However, in the evolutionary algorithm, there are uncertainties in the process of population search and the generation of offspring. Fuzzy set theory has inherent advantages in describing uncertain events and inaccurate information. Fuzzy inference system and hybrid methods with other intelligent computations are widely used in the field of evolutionary optimization, and have shown better results than traditional methods. For example, Melin et al. (Melin et al. 2013) used fuzzy logic to dynamically adjust the weight parameters $\mathrm{C} 1$ and $\mathrm{C} 2$ of the velocity formula in PSO. (Olivas et al. 2017) proposed Ant Colony Optimization (ACO) with interval type-2 fuzzy system, which outperformed a rankbased ACO and ACO using type-1 fuzzy system. (Santiago et al. 2019) proposed a novel MOEA with fuzzy logic based adaptive selection of operators. It identifies which mutation operator is more (or less) promising among simulated binary crossover, uniform mutation, polynomial mutation and DE, for the evolution of the population at each search stage. Also, it uses a fuzzy system to assign the correct application rate to these four operators. Shen et al. (Shen et al. 2019) proposed a multi-objective particle swarm optimization algorithm based on fuzzy optimization, and the experiment has better performance in terms of solution quality, robustness and computational complexity. HSMP (Zou et al. 2020) used the current and past continuous PS centers to automatically establish a T-S fuzzy nonlinear regression prediction model that can predict future PS centers to improve the prediction accuracy when environmental changes occur at the inflection point. (Korashy et al. 2020) proposed a method based on multi-objective grey wolf optimization and fuzzy logic decision-making for solving multi-robot coordination problems and a new objective function to minimize the recognition time between the main and backup relays. The feasibility and effectiveness of this method to solve the coordination problem of DOCRs were discussed on two different systems.

In addition, scholars merge fuzzy systems with machine learning and apply them to the field of multi-objective evolution to improve the performance. For example, (Chen et al. 2018) proposed a hybrid population prediction strategy based on fuzzy inference and one-step prediction. A fuzzy inference model based on the maximum entropy principle is first extracted automatically from the previously found Pareto optimal solution set, and then the trajectory (position and/or direction) of the new Pareto optimal solution set is inferred. This strategy ensures that the algorithm can respond quickly and effectively when the environment changes. Changing PF, thereby, can be traced. (Song et al. 2007) proposed a new fuzzy cognitive map (FCM) learning algorithm based on multi-objective particle swarm optimizations, and experimental results show that the mothed improves the efficiency and robustness of FCMs. In (Yogesh and Ashish 2018), fuzzy logic was used to improve the adaptivity of particle swarm optimization (PSO) by controlling various parameters. Then, the improved PSO was used in K-harmonic means (KHM) for better clustering. (Sankhwar et al. 2019) combined improved grey wolf optimization with fuzzy neural classifier for achieving more accurate financial crisis prediction than other methods.

In this paper, a grey wolf optimization based self-organizing fuzzy multi-objective evolutionary algorithm (GWOSFMEA) is proposed. Fuzzy system is used to dynamically adjust the weighted parameter $\mathrm{F}$ in differential evolution operator, and a new fuzzy differential evolution (FDE) operator is proposed. FDE is used to generate a new initial solution using neighborhood relationship among individuals, followed by polynomial mutation and boundary processing. The new neighborhood relationships of individuals are built by exploiting the peculiarity of the SOM (an unsupervised machine learning method), that is, invariance of the feature distribution, to map the structural information of the current population into Pareto Sets. In addition, in this paper, grey wolf optimization is used to optimize the initial neuron weights of an SOM.

The main features of GWO-SFMEA include:

1) It uses GWO to optimize SOM weights, enabling individuals to search for their neighbors more efficiently in the global scope.

2) When the offspring is generated, a fuzzy inference system is constructed to dynamically adjust the value of the weighting parameter $F$ for high-quality new solutions, and can effectively improve the convergence and diversity of the algorithm.

3) The algorithm utilizes the regular property of multi-objective optimization problems, which can effectively solve functions with complex PF and/or PS shapes.

Experiments on fifteen test problems with complex PF and/or PS shapes were conducted to verify the effectiveness of our proposed algorithm. Results show that its convergence and diversity are better than several state-of-the-art MOEAs.

The remainder of this paper is organized as follows: Section 2 reviews some preliminaries. Section 3 introduces the proposed GWO-SFMEA algorithm in detail. Section 4 presents the test instances and performance metrics. Section 5 describes the parameter settings and experimental results. Section 6 gives additional discussions about GWOSFMEA. Finally, Section 7 draws conclusions. 


\section{Preliminaries}

This paper considers the following form of multi-objective optimization problems (Marler and Arora 2004):

$$
\begin{array}{cl}
\min & F(\boldsymbol{x})=\left(f_{1}(\boldsymbol{x}), f_{2}(\boldsymbol{x}), \ldots, f_{m}(\boldsymbol{x})\right) \\
\text { s.t. } & \boldsymbol{x}=\left(x_{1}, x_{2}, \ldots, x_{n}\right) \in \Omega
\end{array}
$$

where $\Omega$ is the feasible region of the decision space, $\boldsymbol{x}=$ $\left(x_{1}, x_{2}, \ldots, x_{n}\right) \in \Omega$ is the decision variable vector. $n$ is the dimensionality of $\boldsymbol{x}$, and $m$ is the number of objective functions. $F: \Omega \rightarrow \mathbb{R}^{m}$ consists of $m$ objective functions $\left\{f_{i}(\boldsymbol{x})\right\}_{i=1}^{m}$ from the decision space to the objective space.

In this section, we give some knowledge about stochastic operator and grey wolf optimization algorithm.

\subsection{Stochastic operator}

Most MOEAs are optimized by a set of candidate solutions to the target problems. These candidate solutions are generated by random operators. The main difference between MOEAs lies in the properties and search capabilities of the random operators. DE (Price et al. 2005) is one of the most effective operators to solve both single- and multi-objective continuous optimization problems (Li and Zhang 2009; Zhang et al. 2016; Ming et al. 2017; Bošković and Brest 2018).

(Storn 1947; Mendes and Mohais 2005) proposed a variety of differential strategies to implement the mutation operation. Table 1 lists five of them. In $D E / \boldsymbol{x} / \boldsymbol{y} / \boldsymbol{z}, \boldsymbol{x}$ is a variation vector, which can be a random (rand) vector in the population, or the best (best) vector in the current population; $y$ is the number of differential vectors; $\boldsymbol{z}$ represents the mode of crossover, and $\lambda$ the combination factor.

Table 1: Differential Strategies

\begin{tabular}{cc}
\hline Differential strategy & Differential expression \\
\hline $\mathrm{DE} /$ rand/1/bin & $x_{1}+F\left(x_{2}-x_{3}\right)$ \\
$\mathrm{DE} /$ rand/1/bin & $x_{1}+F\left(x_{2}+x_{3}-x_{4}-x_{5}\right)$ \\
$\mathrm{DE} / \mathrm{best} / \mathrm{bin}$ & $x_{b}+F\left(x_{2}-x_{3}\right)$ \\
$\mathrm{DE} / \mathrm{best} / 2 / \mathrm{bin}$ & $x_{b}+F\left(x_{2}+x_{3}-x_{4}-x_{5}\right)$ \\
$\mathrm{DE} /$ rand-to-best/bin & $x_{1}+\lambda\left(x_{b}-x_{i}\right)+F\left(x_{2}-x_{3}\right)$ \\
\hline
\end{tabular}

$D E /$ rand $/ 1 /$ bin and $D E /$ best $/ 2 /$ bin are the most popular and successful differential strategies. This paper adopts the former to ensure the diversity of the population, and then integrates fuzzy inference system into it.

\subsection{Grey wolf optimization (GWO)}

GWO (Mirjalili et al. 2014; Saremi et al. 2015) is a new population intelligence optimization algorithm with fewer parameters, which is simple, flexible, and scalable. It has been widely used in many fields such as machine learning, image processing and so on. For example, Elhariri et al. (Elhariri et al. 2016a;b) successfully applied a GWO-based support vector machine to image classification and EMG signal classification. (Mustaffa et al. 2015) used GWO to optimize the least square support vector machine, and applied it to commodity time series data.

In GWO, grey wolves strictly obey a social dominance hierarchy as shown in Fig. 1, where the $\alpha$ wolf is the leader of the population. The $\beta$ wolf is a candidate of the $\alpha$ wolf, helping the $\alpha$ wolf make decisions or carry out other wolf group activities. The $\delta$ wolf complies with $\alpha$ wolf and $\beta$ wolf, but dominates $\omega$ wolf.

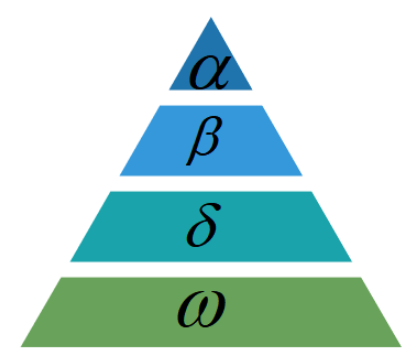

Fig. 1: Hierarchy of grey wolves

In addition to the social hierarchy of wolves, GWO also includes tracking, encircling and attacking prey, etc.

A. Encircling prey

Its mathematical model is:

$\vec{D}=\overrightarrow{\mid C} \cdot \overrightarrow{X_{p}(t)}-\vec{X}(t) \mid$

$\vec{X}(t+1)=\vec{X}_{p}(t)-\vec{A} \cdot \vec{D}$

$\vec{A}=2 \vec{a} \cdot \vec{r}_{1}-\vec{a}$

$\vec{C}=2 \cdot \vec{r}_{2}$

where $t$ indicates the current iteration, $\vec{A}$ and $\vec{C}$ are coefficient vectors, $\vec{X}_{p}$ is the position vector of the prey, and $\vec{X}$ is the position of a grey wolf. In (4), components of $\vec{a}$ are linearly decreased from 2 to 0 over the course of iterations, and $r_{1}$ and $r_{2}$ are random vectors in $[0,1]$.

B. Hunting 
Its mathematical model is:

$\vec{D}_{\alpha}=\left|\vec{C}_{1} \cdot \vec{X}_{\alpha}-\vec{X}\right|, \vec{D}_{\beta}=\left|\vec{C}_{2} \cdot \vec{X}_{\beta}-\vec{X}\right|, \vec{D}_{\delta}=\left|\vec{C}_{3} \cdot \vec{X}_{\delta}-\vec{X}\right|$

$\vec{X}_{1}=\vec{X}_{\alpha}-\vec{A}_{1} \cdot \vec{D}_{\alpha}, \vec{X}_{2}=\vec{X}_{\beta}-\vec{A}_{2} \cdot \vec{D}_{\beta}, \vec{X}_{3}=\vec{X}_{\delta}-\vec{A}_{3} \cdot \vec{D}_{\delta}$

$\vec{X}(t+1)=\frac{\vec{X}_{1}+\vec{X}_{2}+\vec{X}_{3}}{3}$

where $\vec{X}_{\alpha}, \vec{X}_{\beta}$ and $\vec{X}_{\delta}$ represent the position vectors of $\alpha, \beta$ and $\delta$ wolves in the current population, respectively. $\vec{D}_{\alpha}, \vec{D}_{\beta}$ and $\vec{D}_{\delta}$ indicate the distance between candidate wolves in the current population and $\alpha, \beta$, and $\delta$ wolves, respectively.

C. Attacking prey (exploitation)

When the values of $\vec{A}$ are in $[-1,1]$, the next position of the $\omega$ wolf which prepares for attacking the prey can be between its current position and the position of the prey. Otherwise, the wolves will spread out in search of prey for the sake of a global search and avoiding the local optimum.

\section{GWO Based Self-Organizing Fuzzy MOEA (GWO-SFMEA)}

This section proposes a new grey wolf optimization based self-organizing fuzzy multi-objective evolution algorithm. It first uses GWO to optimize the initial network weight vector of the SOM, and then SOM to extract the neighborhood relationship information of the best population individuals. In addition, it uses the fuzzy differential evolution (FDE) operator to generate a new solution.

\subsection{GWO initialized SOM}

SOM network (Kohonen and Teuvo 1990; Teuvo and Kohonen 1998), proposed by Kohonen et al, is an unsupervised machine learning method. The SOM generally consists of two parts: an input layer and an output layer. The neurons between the two layers are fully connected by a network weight vector. It adaptively adjusts this weight vector by detecting the relationship between the input data and the characteristic of the input data. Common topological structure of SOM is one- or two-dimensional. The topological structure of a two-dimensional SOM is shown in Fig. 2(Zhang et al. 2016).

To improve the performance of MOEAs, we utilize GWO to optimize the initial weight vector. Its fitness function is:

$g\left(p_{i}\right)=\frac{100}{F\left(p_{i}\right)}$,

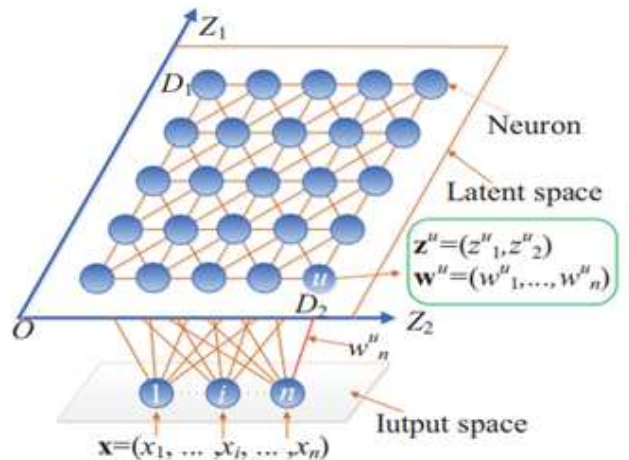

Fig. 2: An illustration of a 2-dimensional SOM

where $F\left(p_{i}\right)=\sum_{n=1}^{N}\left\|x_{q}-c\right\|^{2}$ is used to measure the sum of the Euclidean distance between the input vector and the closest weight vector, and $c=\arg \min _{j}\left\{\left\|x_{q}^{T}-\omega_{j}\right\|^{2}\right\}$. Algorithm 1 shows this optimization process.

\subsection{Fuzzy inference system}

This paper uses Mamdani type fuzzy system (Sivanandam et al. 2007) with triangular membership functions and centroid defuzzification to dynamically adjust the value of the mutation parameter $F$ in the process of generating offspring using the DE operator. This mechanism allows different $F$ values to be applied during each iteration so that the operator can generate higher-quality offspring. The exploration and development capabilities of the algorithm, therefore, can be improved.

During the generation of offsprings, the value of the weighted parameter $F$ is generated by a fuzzy system. The fuzzy system first monitors the search process through the number of iterations and utilization, and then updates the value of the weighted parameter $F$ according to the values of these two variables. The initial utilization value is 1 . The corresponding utilization is decreased by $\frac{1}{\text { controlsize }}$ every time the $F$ value is generated. The value of the controlsize must be carefully tuned. It was set to 500 by trial-and-error.

In Fig. 3 and Fig. 4, we show the linguistic variables with the triangular membership functions for the number of iterations and utilization, respectively.

After the defuzzification process, we obtain a crisp value F. The specific surface function of fuzzy inference system is shown in Fig. 5, and its membership function as shown in Fig. 6.

To model the process with the fuzzy system, we consider 9 rules that help describe the existing relationship between the input and output, as shown in Table 2. 

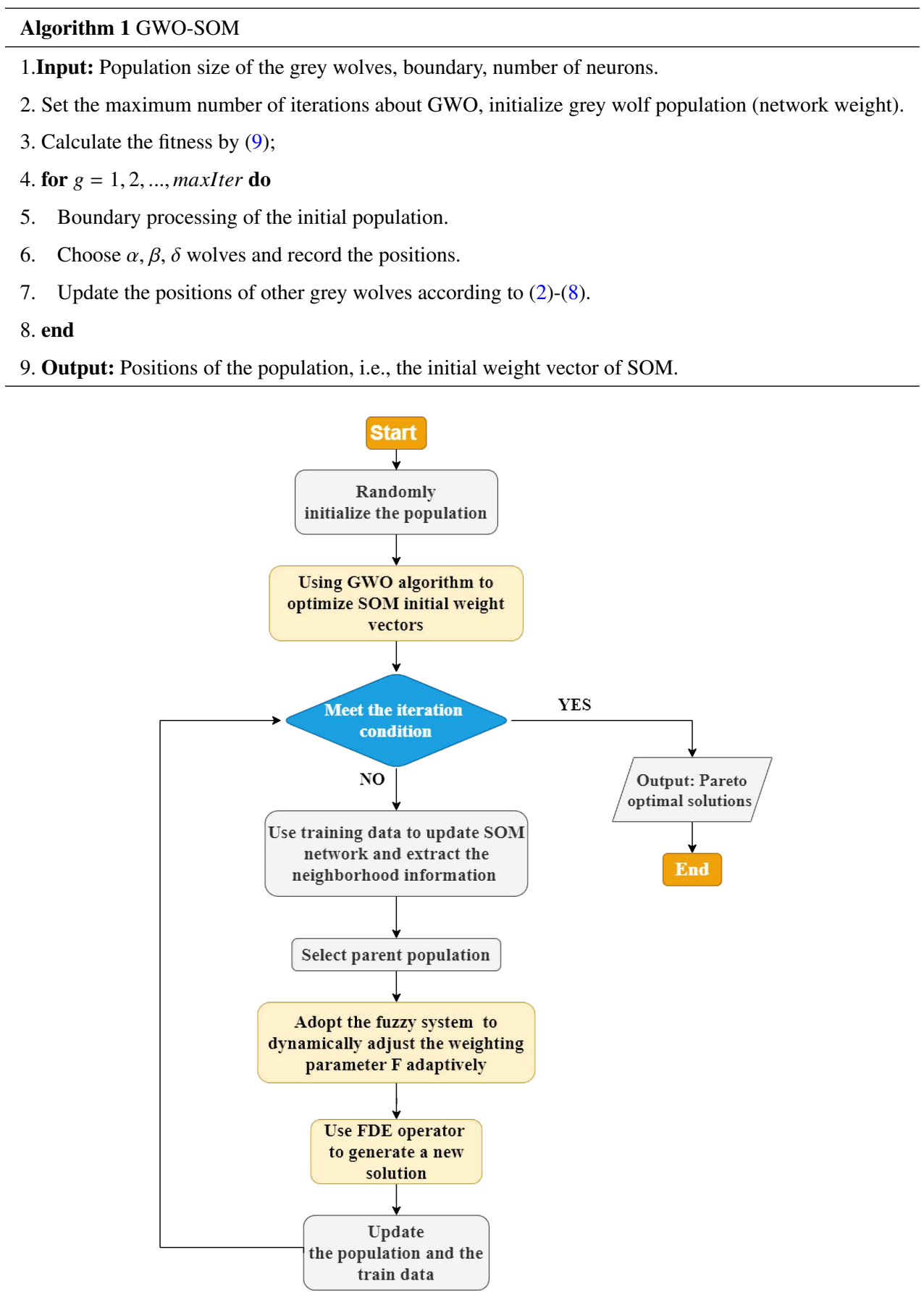

Fig. 7: The GWO-SFMEA framework.

\subsection{GWO-SFMEA Framework}

Fig. 7 shows the specific process of the GWO-SFMEA algorithm.

(1) Randomly initialize the population and set parameters of the proposed algorithm;

(2) Use Algorithm 1 to initialize the weight vectors of the SOM;
(3) Start an iterative loop, use the training data set to update SOM network, i.e., the learning rate, weights, and neighborhood radius. And then extract neighborhood information between the population individuals;

(4) Use the tournament selection mechanism to select the parent population from neighbor or current population;

(5) Use the fuzzy system to dynamically adjust the weighting parameter $\mathrm{F}$ in each iteration. 


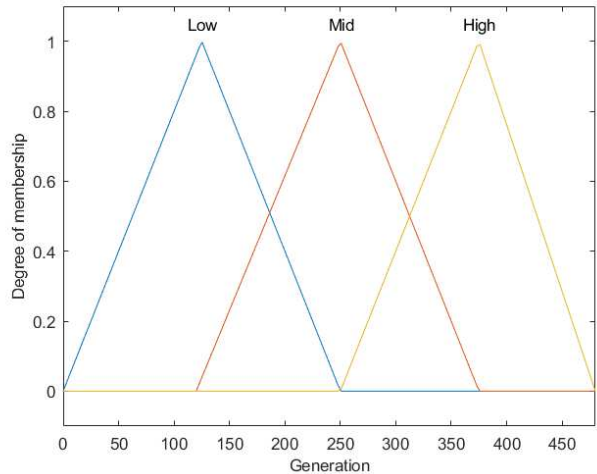

Fig. 3: Generation membership function and the representation of linguistic terms

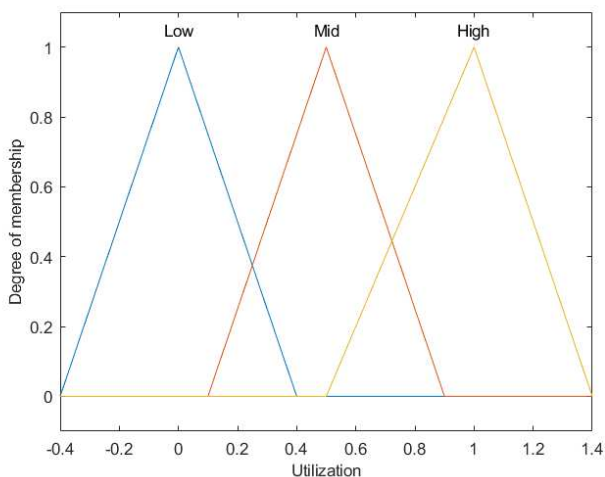

Fig. 4: Utilization membership function and the representation of linguistic terms

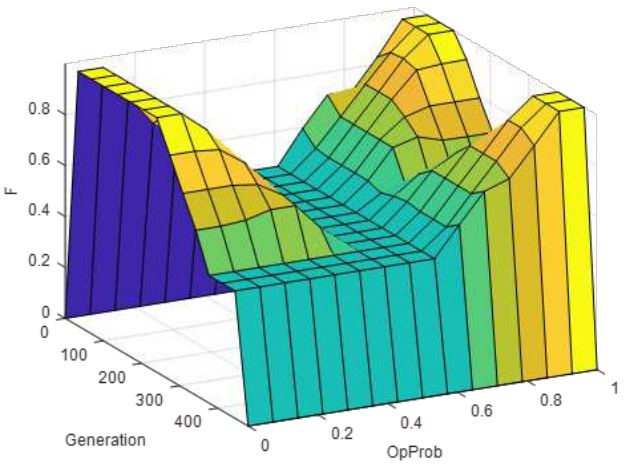

Fig. 5: Surface function graph of fuzzy inference system

(6) Use the FDE operator and the polynomial mutation operator to generate a new solution;

(7) Use the environmental selection mechanism in (Zhang et al. 2016) to update the population and training set. If the termination condition is met, output the optimal solution of the population; otherwise, repeat step (3).

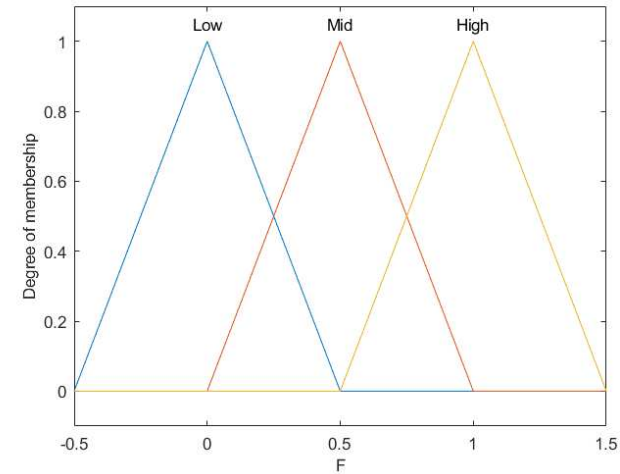

Fig. 6: The membership function and linguistic terms of output F

Table 2: Fuzzy inference rules

Fuzzy inference rules

$R_{1}$ : if Generation is low and utilization is low, then $F$ is high

$R_{2}$ : if Generation is low and utilization is mid, then $F$ is mid

$R_{3}$ : if Generation is low and utilization is high, then $F$ is high

$R_{4}$ : if Generation is mid and utilization is low, then $F$ is high

$R_{5}$ : if Generation is mid and utilization is mid, then $F$ is mid

$R_{6}$ : if Generation is mid and utilization is high, then $F$ is mid

$R_{7}$ : if Generation is high and utilization is low, then $F$ is high

$R_{8}$ : if Generation is high and utilization is mid, then $F$ is mid

$R_{9}$ : if Generation is high and utilization is high, then $F$ is high

\section{Test Instances and Performance Metrics}

In this section, six MOPs with complex PF and PS shapes from (Gu et al. 2012; Zhang et al. 2016) are used as test functions. Table 3 introduces the feasible region of the set of test problems, the number of objective functions, and the dimensionalities of the decision variables.

In order to evaluate the convergence and diversity of the approximate PF obtained by the proposed algorithm, this paper adopts two commonly used performance indicators, i.e., inverted generational distance (IGD) (Zhou et al. 2005; Zhang et al. 2008; Cai et al. 2021) and hypervolume $(H V)$ (Zitzler and Thiele 1999; Zhang et al. 2016).

Let $P F^{*}$ and $P F$ be the evenly distributed Pareto optimal solution set in PF and the obtained non-dominated front respectively. $I G D$ is computed by:

$I G D\left(P F^{*}, P F\right)=\frac{\sum_{x^{*} \in P F^{*}} d\left(x^{*}, P F\right)}{\left|P F^{*}\right|}$,

where $d\left(x^{*}, P F\right)$ is the minimal distance between $x^{*}$ and any point in $P F$, and $\left|P F^{*}\right|$ is the cardinality of $P F^{*}$. When using 
Table 3: The GLT Test Instance Used in the Experiments.

\begin{tabular}{cccl}
\hline Instance & Variable Space & Number of objective functions & Character \\
\hline GLT1 & {$[0,1] \times[-1,1]^{n-1}$} & 2 & Linear and disconnected PF; Nonlinear variable linkage \\
GLT2 & {$[0,1] \times[-1,1]^{n-1}$} & 2 & Convex PF; Nonlinear variable linkage \\
GLT3 & {$[0,1] \times[-1,1]^{n-1}$} & 2 & Convex PF; Nonlinear variable linkage \\
GLT4 & {$[0,1] \times[-1,1]^{n-1}$} & 2 & Convex and disconnected PF; Nonlinear variable linkage \\
GLT5 & {$[0,1]^{2} \times[-1,1]^{n-2}$} & 3 & Convex PF; Nonlinear variable linkage \\
GLT6 & {$[0,1]^{2} \times[-1,1]^{n-2}$} & 3 & Convex and disconnected PF; Nonlinear variable linkage \\
\hline
\end{tabular}

IGD metrics, the true PF value must be known. In the experiments of this section, 1000 uniformly distributed points are selected from $\mathrm{PF}$ to form $\left|P F^{*}\right|$.

The calculation of $H V$ is:

$H V\left(P F, r^{*}\right)=V O L\left(\bigcup_{x \in P F}\left[f_{1}(x), r_{1}^{*}\right] \times \cdots \times\left[f_{m}(x), r_{m}^{*}\right]\right)$,

where $r^{*}=\left(r_{1}^{*}, \ldots, r_{m}^{*}\right)$ is a reference point dominated by any Pareto optimal point in the objective space. $\operatorname{VOL}(\cdot)$ is the Lebesgue measure. In our experiments, we set $r^{*}=(2,2)$ for GLT1 and GLT3, $r^{*}=(2,11)$ for GLT2, $r^{*}=(2,3)$ for GLT4, $r^{*}=(2,2,2)$ for GLT5 and GLT6. Both the IGD and $H V$ metrics measure the population convergence and diversity. The smaller (larger) the value of $\operatorname{IGD}(H \mathrm{~V})$ is, the better the performance of an algorithm.

\section{Experimental Results}

In this section, the proposed algorithm GWO-SFMEA compares with SMEA(Zhang et al. 2016), MOEA/D-DE(Li and Zhang 2009), SOM-NSGA-II(Norouzi and Rakhshandehroo 2011), SMPSO(Jing et al. 2019) and FAME(Santiago et al. 2019) in the GLT test instances with regard to IGD value and $H V$ value.

\subsection{Parameter setting}

The proposed algorithm is implemented on Windows 10, 64-bit, MATLAB2018a. The detailed parameters are set as follows:

- SOM structures: 1-dimensional structure $1 \times 100$ for biobjective MOPs, 2-dimensional $7 \times 15$ for tri-objective MOPs; initial learning rate $\tau_{0}=0.7$;

- Size of neighborhood mating pools: $H=5$;

- Probability of mating restriction: $\beta=0.9$;

- Control parameters for FDE operator: $C R=1$;

- Control parameters for PM: $p_{m}=\frac{1}{n}, \eta_{m}=20$.

The implementation and parameter setting of other algorithms were configured according to the suggestions of the original papers. Table 4 summarizes these parameters.

\subsection{Analysis of results}

\section{A. Time comparison}

Table 5 shows the average time of SMEA and the proposed algorithm on 30 executions of 6 multi-objective test problems. Their time complexity on the bi-objective problem is similar, our proposed algorithm is faster on the triobjective problem.

Table 5: Average time of SMEA and GWO-SFMEA on the GLT test suite / $s$

\begin{tabular}{lcc}
\hline Instance & SMEA & GWO-SFMEA \\
\hline GLT1 & 108.83 & 108.76 \\
GLT2 & 104.47 & 98.67 \\
GLT3 & 97.41 & 99.52 \\
GLT4 & 97.72 & 99.19 \\
GLT5 & 217.31 & 192.90 \\
GLT6 & 214.44 & 204.19 \\
\hline
\end{tabular}

\section{B. Comparison of IGD and HV value}

The mean and standard deviation of the IGD and HV values of the 30 final populations generated by the six algorithms on the GLT test function are given in Table 6. The gray background indicates the best results (minimum IGD or maximum $H V)$; "§", "\" and " " in the table indicate that the performance of the algorithm GWO-SFMEA is better than, worse than, and similar to that of the comparison algorithm, respectively. Generally, the performance of GWOSFMEA was significantly better than other algorithms on all GLT issues.

In terms of $I G D$, the value achieved by the the proposed algorithm was lower than the other five algorithms in both bi-objective and tri-objective problems. The performance of SMPSO on GLT1 and GLT3 was inferior to all algorithms. MOEA/D-DE performed the worst on GLT2, GLT5 and GLT6. The value obtained by FAME on GLT4 was inferior to other algorithms. 
Table 4: Parameters settings

\begin{tabular}{|c|c|c|}
\hline Algorithm & SOM-NSGA-II & SMEA \\
\hline population size & 100/105 for bi/tri-objective MOPs & 100/105 for bi/tri-objective MOPs \\
\hline SOM structures & $1 \times 5 / 3 \times 5$ for bi/tri-objective MOPs & $1 \times 100 / 7 \times 15$ for bi/tri-objective MOPs \\
\hline Differential evolution & $\mathrm{F}=0.3, \mathrm{CR}=1.0$ & $\mathrm{~F}=0.9, \mathrm{CR}=1.0$ \\
\hline Polynomial evolution & $p_{m}=1 / n, \eta_{m}=20$ & $p_{m}=1 / n, \eta_{m}=20$ \\
\hline Neighborhood size & & 5 , probability of mating restriction: 0.9 \\
\hline Algorithm & FAME & SMPSO \\
\hline population size & 25/100 for bi/tri-objective MOPs & $100 / 150$ for bi/tri-objective MOPs \\
\hline Archive size & $100 / 200$ for bi/tri-objective MOPs & $100 / 150$ for bi/tri-objective MOPs \\
\hline SOM structures & & $1 \times 100 / 15 \times 10$ for bi/tri-objective MOPs \\
\hline Recombination & $\mathrm{DE}: \mathrm{F}=0.5, \mathrm{CR}=1.0, \mathrm{SBX}: \eta_{c}=20$ & \\
\hline Polynomial evolution & $p_{m}=0.3, \eta_{m}=20$ & \\
\hline Uniform mutation & $p_{m}=0.3$ & \\
\hline Algorithm & MOEA/D-DE & \\
\hline population size & 100/105 for bi/tri-objective MOPs & \\
\hline Differential evolution & $\mathrm{F}=0.9, \mathrm{CR}=0.8$ & \\
\hline Polynomial evolution & $p_{m}=1 / n, \eta_{m}=20$ & \\
\hline Neighborhood size & 5, probability of mating restriction: 0.9 & \\
\hline
\end{tabular}

With regard to $H V$, the proposed algorithm obtained better values than other algorithms on all GLT test instances except GLT1 and GLT3, where FAME and SMPSO ranked first respectively. However, FAME and MOEA/D-DE performed the worst on bi-objective problems(GLT4 for FAME, GLT2 and GLT3 for MOEA/D-DE). SMPSO had worst performance on tri-objective problem(GLT5 and GLT6).

Table 7 shows the Friedman ranks of the six algorithm$\mathrm{s}$ for the two considered indicators, with $95 \%$ significance. Regardless of $I G D$ or $H V$, the average ranking of the proposed algorithm was the first, followed by SMEA. MOEA/DDE ranked last on all metric values, which means it has the worst performance.

Table 7: Average ranking of the algorithms.

\begin{tabular}{l|cc}
\hline Algorithm & $H V$ Rank & IGD Rank \\
\hline SOM-NSGA-II & 4.33 & 4.00 \\
FAME & 3.83 & 4.33 \\
MOEA/D-DE & 4.50 & 5.17 \\
SMEA & 2.67 & 2.00 \\
SMPSO & 4.17 & 4.50 \\
GWO-SFMEA & 1.50 & 1.00 \\
\hline
\end{tabular}

C. Population distribution graph
In order to further compare SMEA, FAME and GWOSFMEA, Fig. 8 shows the distribution of the final population implemented independently by three algorithms for 30 times.

For GLT1, the distribution of the final population achieved by FAME is better than SMEA and GWO-SFMEA, which is consistent with the optimal performance of $H V$ of FAME in Table 6. Besides, the population distribution generated by GWO-SFMEA is better than SMEA. Both FAME and the proposed GWO-SFMEA apply fuzzy systems and outperform SMEA, indicating that the use of fuzzy systems in EAs can make the population distribution more unifor$\mathrm{m}$. The population distributions generated by SMEA, FAME and GWO-SFMEA on GLT2 can cover the whole PFs well and do not show big difference. SMEA is not much different from GWO-SFMEA on GLT3, but these two algorithms are better than FAME. The distribution of the population achieved by FAME, the right half part is not evenly distributed. Although the FAME guarantees the diversity of the population on GLT4, its distribution is uneven. By contrast, GWO-SFMEA guarantees the uniformity. GWOSFMEA performs better than the SEMA and FAME on triobjective problems (GLT5 and GLT6).

By analyzing the above experimental results, we can found the SMPSO and MOEA/D-DE performed worst on both complicated PF or PS shapes, which might be because the particles have poor search ability or the set of the weight vectors. FAME works well on MOPs with three objectives, 

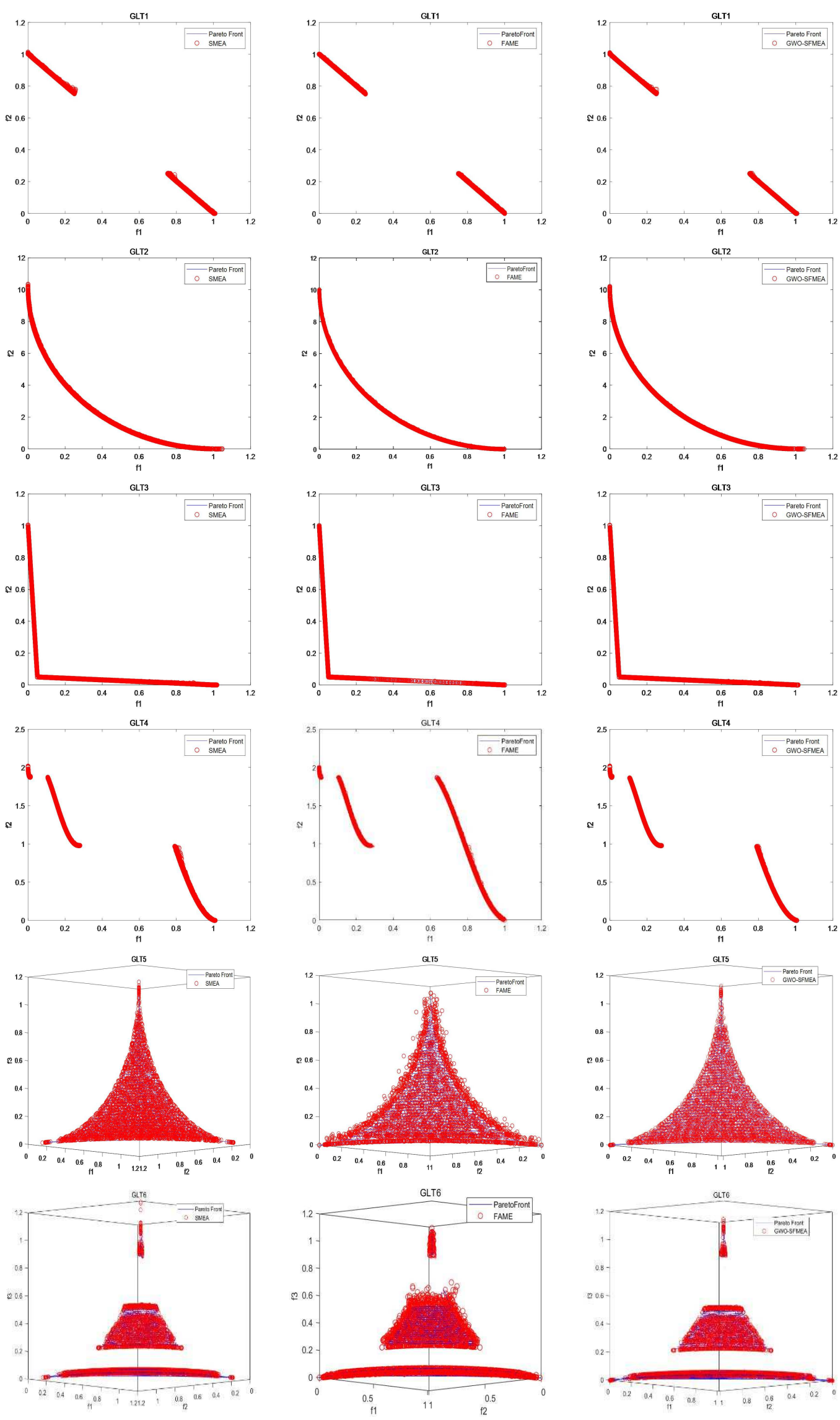

Fig. 8: The final populations in the objective space obtained by SMEA, FAME and GWO-SFMEA on GLT1-GLT6 over 30 runs. 
Table 6: Statistical result (Mean Std [Rank]) of the six algorithms on the GLT test suite of the IGD and $H V$ metrics.

\begin{tabular}{|c|c|c|c|c|c|c|c|}
\hline \multicolumn{2}{|c|}{ Instance } & SOM-NSGA-II & FAME & MOEA/D-DE & SMEA & SMPSO & GWO-SFMEA \\
\hline \multicolumn{8}{|c|}{$I G D$} \\
\hline \multirow[t]{2}{*}{ GLT1 } & mean & $4.437 \mathrm{E}-03 \S[4]$ & $4.140 \mathrm{E}-03 \S[3]$ & $5.522 \mathrm{E}-03 \S[5]$ & $2.762 \mathrm{E}-03 \S[2]$ & $2.38 \mathrm{E}-02 \S[6]$ & $2.107 \mathrm{E}-03[1]$ \\
\hline & std & $(2.239 \mathrm{E}-03)$ & (3.230E-03) & $(2.681 \mathrm{E}-04)$ & (5.173E-04) & $(2.25 \mathrm{E}-03)$ & (5.212E-05) \\
\hline \multirow[t]{2}{*}{ GLT2 } & mean & $4.345 \mathrm{E}-02 \S[4]$ & $6.209 \mathrm{E}-02 \S[5]$ & $3.579 \mathrm{E}-01 \S[6]$ & $3.765 \mathrm{E}-02 \S[2]$ & $3.82 \mathrm{E}-02 \S[3]$ & $3.699 \mathrm{E}-02[1]$ \\
\hline & std & (3.080E-03) & $(2.481 \mathrm{E}-02)$ & $(5.802 \mathrm{E}-02)$ & (2.004E-03) & (1.88E-03) & (1.793E-03) \\
\hline \multirow[t]{2}{*}{ GLT3 } & mean & $8.704 \mathrm{E}-03 \S[3]$ & $4.790 \mathrm{E}-02 \S[5]$ & $2.348 \mathrm{E}-02 \S[4]$ & $5.756 \mathrm{E}-03 \S[2]$ & $9.32 \mathrm{E}-02 \S[6]$ & $5.165 \mathrm{E}-03[1]$ \\
\hline & std & (4.219E-03) & $(1.624 \mathrm{E}-02)$ & (3.311E-03) & $(1.296 \mathrm{E}-03)$ & (5.32E-03) & (5.900E-04) \\
\hline \multirow[t]{2}{*}{ GLT4 } & mean & $1.769 \mathrm{E}-02 \S[3]$ & $1.475 \mathrm{E}-01 \S[6]$ & $2.162 \mathrm{E}-02 \S[4]$ & $6.752 \mathrm{E}-03 \S[2]$ & $4.17 \mathrm{E}-02 \S[5]$ & $5.876 \mathrm{E}-03[1]$ \\
\hline & std & $(2.702 \mathrm{E}-02)$ & $(1.267 \mathrm{E}-01)$ & (3.928E-03) & $(1.835 \mathrm{E}-02)$ & (4.90E-03) & (1.258E-04) \\
\hline \multirow[t]{2}{*}{ GLT5 } & mean & $5.530 \mathrm{E}-02 \S[5]$ & $3.233 \mathrm{E}-02 \S[3]$ & $8.278 \mathrm{E}-02 \S[6]$ & $2.947 \mathrm{E}-02 \S[2]$ & $4.77 \mathrm{E}-02 \S[4]$ & $2.933 \mathrm{E}-02[1]$ \\
\hline & std & (3.843E-04) & (5.200E-04) & (2.006E-03) & (3.724E-04) & (2.57E-03) & (3.656E-04) \\
\hline \multirow[t]{2}{*}{ GLT6 } & mean & $5.123 \mathrm{E}-02 \S[5]$ & $4.810 \mathrm{E}-02 \S[4]$ & $5.149 \mathrm{E}-02 \S[6]$ & $2.163 \mathrm{E}-02 \S[2]$ & $4.12 \mathrm{E}-02 \S[3]$ & $2.159 \mathrm{E}-02[1]$ \\
\hline & std & (8.447E-03) & (5.266E-03) & (1.295E-03) & (3.489E-04) & (3.34E-03) & (2.193E-04) \\
\hline \multicolumn{8}{|c|}{$H V$} \\
\hline \multirow[t]{2}{*}{ GLT1 } & mean & $3.357 \mathrm{E}+00 \S[5]$ & $3.377 \mathrm{E}+00 \backslash[1]$ & $3.369 \mathrm{E}+00 \sim[2]$ & $3.366 \mathrm{E}+00 \S[4]$ & $3.31 \mathrm{E}+00 \S[6]$ & $3.369 \mathrm{E}+00[3]$ \\
\hline & std & (3.361E-03) & (9.401E-02) & $(6.176-04)$ & (3.361E-03) & (5.30E-03) & $(1.100 \mathrm{E}-03)$ \\
\hline \multirow[t]{2}{*}{ GLT2 } & mean & $1.975 \mathrm{E}+01 \S[5]$ & $1.976 \mathrm{E}+01 \S[4]$ & $1.942 \mathrm{E}+01 \S[6]$ & $1.978 \mathrm{E}+01 \S[3]$ & $1.98 \mathrm{E}+01 \sim[2]$ & $1.980 \mathrm{E}+01[1]$ \\
\hline & std & (4.990E-03) & $(1.504 \mathrm{E}-02)$ & $(5.492 \mathrm{E}-02)$ & $(5.825 \mathrm{E}-03)$ & (1.01E-02) & (2.032E-03) \\
\hline \multirow[t]{2}{*}{ GLT3 } & mean & $3.947 \mathrm{E}+00 \S[4]$ & $3.939 \mathrm{E}+00 \S[6]$ & $3.943 \mathrm{E}+00 \S[5]$ & $3.948 \mathrm{E}+00 \S[3]$ & $3.95 \mathrm{E}+00 \sim[1]$ & $3.949 \mathrm{E}+00[2]$ \\
\hline & std & $(1.024 \mathrm{E}-03)$ & (3.520E-03) & (6.799E-04) & $(4.186 \mathrm{E}-04)$ & (1.06E-03) & (2.934E-04) \\
\hline \multirow[t]{2}{*}{ GLT4 } & mean & $4.979 \mathrm{E}+00 \S[3]$ & $4.613 \mathrm{E}+00 \S[6]$ & $4.969 \mathrm{E}+00 \S[5]$ & $4.988 \mathrm{E}+00 \S[2]$ & $4.97 \mathrm{E}+00 \S[4]$ & $4.992 \mathrm{E}+00[1]$ \\
\hline & std & $(2.934 \mathrm{E}-02)$ & (3.970E-01) & (8.920E-03) & $(3.482 \mathrm{E}-02)$ & (2.59E-02) & (5.912E-04) \\
\hline \multirow[t]{2}{*}{ GLT5 } & mean & $7.947 \mathrm{E}+00 \S[4]$ & $7.964 \mathrm{E}+00 \S[3]$ & $7.940 \mathrm{E}+00 \S[5]$ & $7.969 \mathrm{E}+00 \sim[2]$ & $3.90 \mathrm{E}+00 \S[6]$ & $7.969 \mathrm{E}+00[1]$ \\
\hline & std & $(2.239 \mathrm{E}-03)$ & $(5.000 \mathrm{E}-04)$ & (1.297E-03) & (1.617E-04) & $(1.56 \mathrm{E}-02)$ & (9.977E-05) \\
\hline \multirow[t]{2}{*}{ GLT6 } & mean & $7.935 \mathrm{E}+00 \S[5]$ & $7.945 \mathrm{E}+00 \S[3]$ & $7.940 \mathrm{E}+00 \S[4]$ & $7.962 \mathrm{E}+00 \sim[2]$ & $3.86 \mathrm{E}+00 \S[6]$ & $7.962 \mathrm{E}+00[1]$ \\
\hline & std & $(6.048 \mathrm{E}-03)$ & $(2.852 \mathrm{E}-02)$ & (3.323E-03) & (3.044E-04) & $(1.37 \mathrm{E}-02)$ & (2.382E-04) \\
\hline
\end{tabular}

but it performs poorly on the GLT3 might be due to the random selection of the operator. SOM-NSGA-II and SMEA employs SOM to extract neighborhood information of the population, therefore, they can construct better quality parent population. Similarly, the algorithm proposed in this paper uses SOM to construct parent population, and uses the fuzzy system to dynamically adjust the parameters in the generation of its offspring to ensure that each iteration can produce high-quality solutions. Thus, GWO-SFMEA has a significant advantage in dealing with these instances.

\section{Discussions}

\subsection{Test Function with Complex PF Shapes}

The WFG(Huband et al. 2005) test instances have a complex PF shape and a simple PS shape. Its characteristics are shown in Table 8.

Through preliminary experiments, the output surface of the fuzzy system with the mutation parameter F in the GWOSFMEA algorithm is shown in Fig. 9, $\tau_{0}=0.9, C R=0.8$ and the values of other parameters are the same as in Section 5.1 .

The five algorithms were run on these instances 30 times. The mean and standard deviation of the $I G D$ and $H V$ values of the final population are shown in Table 9. The reference point for the bi-objective WFG that is used to calculate the $H V$ metric is $r^{*}=(3,5)$. 
Table 9: Statistical Result (Mean Std [Rank]) of The Five Algorithms on the GLT Test Suite of the IGD and $H V$ Metrics.

\begin{tabular}{|c|c|c|c|c|c|c|}
\hline \multicolumn{2}{|l|}{ Instance } & SOM-NSGA-II & FAME & MOEA/D-DE & SMEA & GWO-SFMEA \\
\hline \multicolumn{7}{|c|}{$I G D$} \\
\hline \multirow{2}{*}{ WFG1 } & mean & $1.433 \mathrm{E}+00 \S[3]$ & $1.491 \mathrm{E}-02 \backslash[1]$ & $1.695 \mathrm{E}+00 \S[5]$ & $1.565 \mathrm{E}+00 \S[4]$ & $1.085 \mathrm{E}+00[2]$ \\
\hline & std & (7.333E-02) & $(1.308 \mathrm{E}-03)$ & $(1.122 \mathrm{E}-02)$ & $(9.525 \mathrm{E}-02)$ & $(2.019 \mathrm{E}-02)$ \\
\hline \multirow[t]{2}{*}{ WFG2 } & mean & $1.433 \mathrm{E}-02 \S[2]$ & $1.846 \mathrm{E}-02 \S[4]$ & $3.790 \mathrm{E}-02 \S[5]$ & $1.474 \mathrm{E}-02 \S[3]$ & $1.121 \mathrm{E}-02[1]$ \\
\hline & std & (6.093E-03) & (3.859E-02) & (6.109E-04) & $(7.078 \mathrm{E}-04)$ & (4.670E-04) \\
\hline \multirow[t]{2}{*}{ WFG3 } & mean & $1.450 \mathrm{E}-01 \S[5]$ & $1.127 \mathrm{E}-02 \backslash[1]$ & $1.420 \mathrm{E}-01 \S[4]$ & $1.388 \mathrm{E}-01 \S[3]$ & $1.137 \mathrm{E}-02[2]$ \\
\hline & std & (6.990E-04) & (6.682E-05) & (3.025E-04) & (1.380E-04) & (7.061E-05) \\
\hline \multirow[t]{2}{*}{ WFG4 } & mean & $6.037 \mathrm{E}-02 \S[4]$ & $1.084 \mathrm{E}-02 \backslash[1]$ & $5.025 \mathrm{E}-02 \S[3]$ & $7.784 \mathrm{E}-02 \S[5]$ & $3.059 \mathrm{E}-02[2]$ \\
\hline & std & (5.681E-03) & (1.364E-04) & (6.321E-03) & (6.902E-03) & (3.959E-02) \\
\hline \multirow[t]{2}{*}{ WFG5 } & mean & $6.902 \mathrm{E}-02 \S[5]$ & $6.638 \mathrm{E}-02 \S[2]$ & $6.747 \mathrm{E}-02 \S[4]$ & $6.663 \mathrm{E}-02 \S[3]$ & $6.467 \mathrm{E}-02[1]$ \\
\hline & std & $(5.246 \mathrm{E}-04)$ & (4.037E-05) & $(4.476 \mathrm{E}-04)$ & $(1.138 \mathrm{E}-04)$ & (5.701E-03) \\
\hline \multirow[t]{2}{*}{ WFG6 } & mean & $3.334 \mathrm{E}-01 \S[4]$ & $1.288 \mathrm{E}-02 \backslash[1]$ & $3.352 \mathrm{E}-01 \S[5]$ & $3.265 \mathrm{E}-01 \S[3]$ & $3.022 \mathrm{E}-01[2]$ \\
\hline & std & (4.137E-03) & (2.792E-04) & $(2.240 \mathrm{E}-02)$ & $(2.090 \mathrm{E}-02)$ & (3.069E-04) \\
\hline \multirow[t]{2}{*}{ WFG7 } & mean & $2.994 \mathrm{E}-02 \S[5]$ & $1.261 \mathrm{E}-02 \S[3]$ & $1.815 \mathrm{E}-02 \S[4]$ & $1.144 \mathrm{E}-02 \S[2]$ & $1.071 \mathrm{E}-02[1]$ \\
\hline & std & (1.527E-03) & $(2.062 \mathrm{E}-04)$ & $(2.968 \mathrm{E}-04)$ & $(2.526 \mathrm{E}-04)$ & (3.107E-04) \\
\hline \multirow[t]{2}{*}{ WFG8 } & mean & $5.755 \mathrm{E}-02 \S[4]$ & $1.033 \mathrm{E}-01 \S[5]$ & $3.989 \mathrm{E}-02 \S[3]$ & $2.666 \mathrm{E}-02 \S[2]$ & $1.186 \mathrm{E}-02[1]$ \\
\hline & std & (6.643E-03) & $(2.750 \mathrm{E}-02)$ & (7.611E-03) & (6.924E-03) & (2.819E-04) \\
\hline \multirow[t]{2}{*}{ WFG9 } & mean & 2.707E-01§[5] & $1.448 \mathrm{E}-02 \backslash[1]$ & $2.435 \mathrm{E}-01 \S[3]$ & $2.561 \mathrm{E}-01 \S[4]$ & $1.984 \mathrm{E}-01[2]$ \\
\hline & std & $(1.217 \mathrm{E}-02)$ & $(4.970 \mathrm{E}-03)$ & $(3.200 \mathrm{E}-02)$ & $(2.980 \mathrm{E}-02)$ & $(7.404 \mathrm{E}-04)$ \\
\hline \multicolumn{7}{|c|}{$H V$} \\
\hline \multirow[t]{2}{*}{ WFG1 } & mean & $4.428 \mathrm{E}+00 \S[4]$ & $1.207 \mathrm{E}+01 \backslash[1]$ & $5.809 \mathrm{E}+00 \S[3]$ & $3.873 \mathrm{E}+00 \S[5]$ & $5.878 \mathrm{E}+00[2]$ \\
\hline & std & (2.667E-01) & $(4.180 \mathrm{E}-03)$ & $(5.017-02)$ & (3.781E-01) & $(1.217 \mathrm{E}-01)$ \\
\hline \multirow[t]{2}{*}{ WFG2 } & mean & $1.141 \mathrm{E}+01 \S[3]$ & $1.136 \mathrm{E}+01 \S[5]$ & $1.143 \mathrm{E}+01 \S[2]$ & $1.141 \mathrm{E}+01 \S[4]$ & $1.145 \mathrm{E}+01[1]$ \\
\hline & std & (3.455E-03) & $(4.195 \mathrm{E}-01)$ & (1.397E-03) & $(6.347 \mathrm{E}-03)$ & (5.476E-02) \\
\hline \multirow[t]{2}{*}{ WFG3 } & mean & $1.089 \mathrm{E}+01 \S[5]$ & $1.096 \mathrm{E}+01 \sim[2]$ & $1.091 \mathrm{E}+01 \S[4]$ & $1.594 \mathrm{E}+01 \backslash[1]$ & $1.096 \mathrm{E}+01[3]$ \\
\hline & std & (5.643E-03) & $(2.100 \mathrm{E}-04)$ & (3.319E-03) & $(1.946 \mathrm{E}-03)$ & $(1.141 \mathrm{E}-03)$ \\
\hline \multirow[t]{2}{*}{ WFG4 } & mean & $8.328 \mathrm{E}+00 \S[4]$ & $8.684 \mathrm{E}+00 \backslash[1]$ & $8.443 \mathrm{E}+00 \S[3]$ & $8.204 \mathrm{E}+00 \S[5]$ & $8.559 \mathrm{E}+00[2]$ \\
\hline & std & (3.591E-02) & $(1.710 \mathrm{E}-03)$ & $(3.884 \mathrm{E}-02)$ & $(3.966 \mathrm{E}-02)$ & $(1.993 \mathrm{E}-02)$ \\
\hline \multirow[t]{2}{*}{ WFG5 } & mean & $8.158 \mathrm{E}+00 \S[4]$ & $8.181 \mathrm{E}+00 \S[3]$ & $8.138 \mathrm{E}+00 \S[5]$ & $8.233 \mathrm{E}+00 \S[2]$ & $8.251 \mathrm{E}+00[1]$ \\
\hline & std & $(5.498 \mathrm{E}-02)$ & (3.902E-02) & $(3.069 \mathrm{E}-02)$ & $(4.557 \mathrm{E}-02)$ & $(3.188 \mathrm{E}-02)$ \\
\hline \multirow[t]{2}{*}{ WFG6 } & mean & $6.344 \mathrm{E}+00 \S[4]$ & $8.677 \mathrm{E}+00 \backslash[1]$ & $6.337 \mathrm{E}+00 \S[5]$ & $6.383 \mathrm{E}+00 \S[3]$ & $6.527 \mathrm{E}+00[2]$ \\
\hline & std & (2.199E-03) & (3.651E-02) & $(1.139 \mathrm{E}-01)$ & (1.064E-01) & (3.112E-04) \\
\hline \multirow[t]{2}{*}{ WFG7 } & mean & $8.575 \mathrm{E}+00 \S[5]$ & $8.686 \mathrm{E}+00 \S[2]$ & $8.644 \mathrm{E}+00 \S[4]$ & $8.671 \mathrm{E}+00 \S[3]$ & $8.687 \mathrm{E}+00[1]$ \\
\hline & std & (7.901E-03) & $(2.620 \mathrm{E}-03)$ & (3.616E-03) & $(2.129 \mathrm{E}-03)$ & $(2.600 \mathrm{E}-04)$ \\
\hline \multirow[t]{2}{*}{ WFG8 } & mean & $8.352 \mathrm{E}+00 \S[4]$ & $7.049 \mathrm{E}+00 \S[5]$ & $8.482 \mathrm{E}+00 \S[3]$ & $8.528 \mathrm{E}+002 \S[2]$ & $8.675 \mathrm{E}+00[1]$ \\
\hline & std & (3.872E-02) & $(4.843 \mathrm{E}-01)$ & $(4.711 \mathrm{E}-02)$ & $(4.456 \mathrm{E}-02)$ & $(2.495 \mathrm{E}-03)$ \\
\hline \multirow[t]{2}{*}{ WFG9 } & mean & $6.102 \mathrm{E}+00 \S[5]$ & $8.448 \mathrm{E}+00 \backslash[1]$ & $6.179 \mathrm{E}+00 \S[3]$ & $6.174 \mathrm{E}+00 \S[4]$ & $6.451 \mathrm{E}+00[2]$ \\
\hline & std & $(6.438 \mathrm{E}-02)$ & $(4.316 \mathrm{E}-02)$ & $(1.563 \mathrm{E}-01)$ & $(1.518 \mathrm{E}-01)$ & $(2.543 \mathrm{E}-02)$ \\
\hline
\end{tabular}


Table 8: The WFG test instance used in the experiments.

\begin{tabular}{ll}
\hline Instance & Character \\
\hline WFG1 & separable; Uni-modal \\
WFG2 & non-separable; Uni-modal \\
WFG3 & non-separable; Uni-modal \\
WFG4 & separable; multi-modal \\
WFG5 & separable; deceptive \\
WFG6 & non-separable; Uni-modal \\
WFG7 & Separate; Uni-modal \\
WFG8 & non-separable; Uni-modal \\
WFG9 & non-separable; multi-modal; deceptive \\
\hline
\end{tabular}

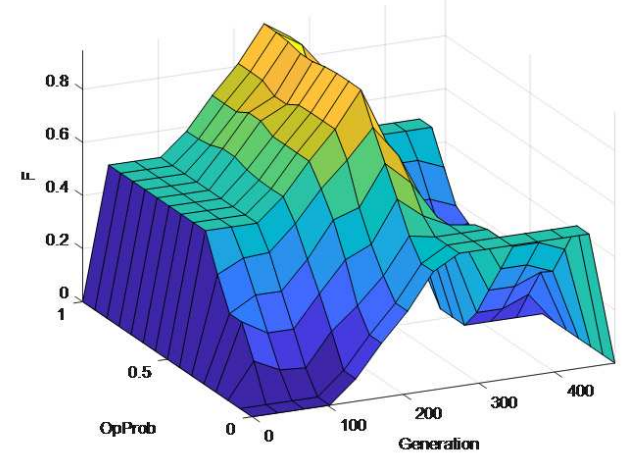

Fig. 9: Output surface of fuzzy system with weighted parameter $\mathrm{F}$.

As show in Table 9, the proposed algorithm achieved 8 best values on 18 average metric values. In terms of $I G D$, the proposed algorithm obtained lower values than other algorithms on WFG2, WFG5, WFG7 and WFG8. FAME performed best on the rest of problems. MOEA/D-DE and SOMNSGA-II obtained worst values on WFG1, WFG2, WFG6 and WFG3, WFG5, WFG9 respectively.

With regard to $H V$, the proposed algorithm was better than the other algorithm on WFG2, WFG5, WFG7-8. It was inferior to FAME and SMEA on WFG1, WFG4, WFG6, WFG9 and WFG3 respectively. However, FAME and SMEA obtain worst value on WFG8 and WFG1 respectively. MOEA /D-DE and SOM-NSGA-II obtained worst values on WFG56 and WFG3, WFG7, WFG9, respectively.

In conclusion, GWO-SFMEA achieved good performance on WFG2, WFG5, WFG7, and WFG8 and ranked second on other problems. It can be seen that the algorithm proposed in this paper is able to tackle MOPs with complex PF shape.

Table 10 shows the Friedman ranks of the five algorithms for the two considered indicators, with $95 \%$ significance. The proposed algorithm achieved higher rank than other algorithms.
Table 10: Average Rankings of the Algorithms.

\begin{tabular}{l|cc}
\hline Algorithm & $H V$ Rank & $I G D$ Rank \\
\hline SOM-NSGA-II & 4.22 & 4.11 \\
FAME & 2.33 & 2.11 \\
MOEA/D-DE & 3.56 & 4.00 \\
SMEA & 3.22 & 3.22 \\
GWO-SFMEA & 1.67 & 1.56 \\
\hline
\end{tabular}

\subsection{Application of fuzzy system}

In order to study whether fuzzy system improves the convergence of the algorithm and the diversity of the population, the GWO-SFMEA was compared with the version without fuzzy inference system by running two algorithms independently on the GLT problems for 30 times. The average $I G D$ and $H V$ values after the implementation of two algorithms are shown in Fig. 10. The larger the $H V$ value is or the smaller the $I G D$ value is, the better performance of the algorithm is.

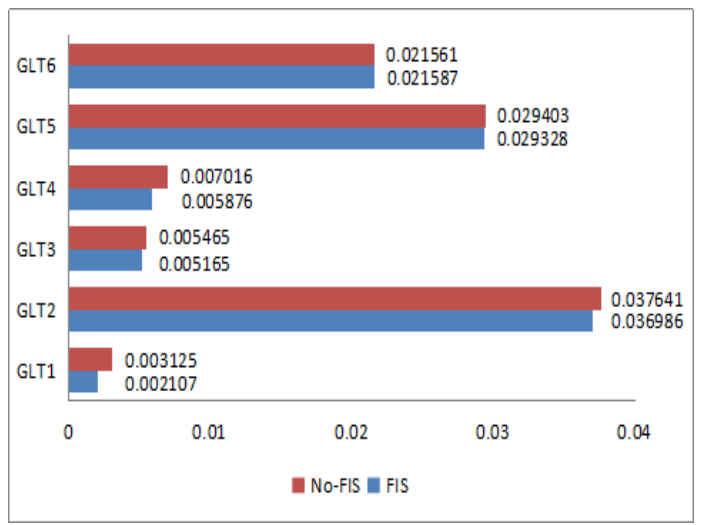

(a) The impact of fuzzy system on $I G D$

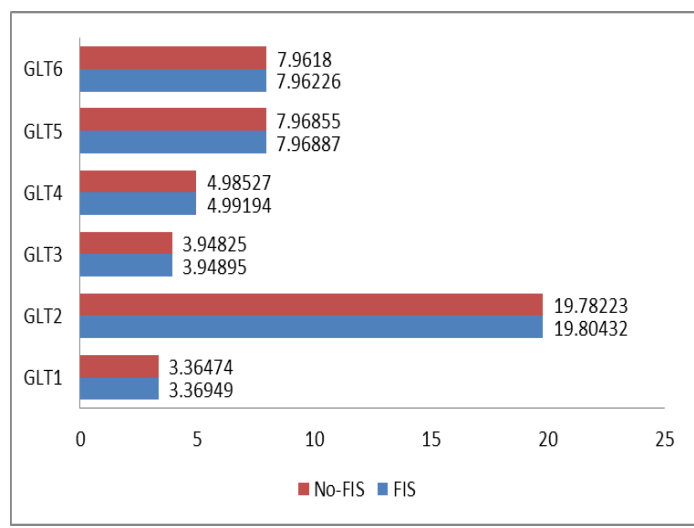

(b) The impact of fuzzy system on $H V$

Fig. 10: The impact of fuzzy system on $I G D$ and $H V$. 
It can be seen apparently from Fig. 10 that the $H V$ value increased, but its corresponding $I G D$ decreased at the same time on GLT1-GLT5 after using fuzzy system; On the GLT6, the $H V$ and $I G D$ both increased, but the increment of accuracy of $I G D$ was not large.

In summary, the performance of the propose algorithm on all instances was improved after adopting the fuzzy inference system, which shows that well-organized fuzzy inference system in the MOEAs can improve the performance of the algorithm and can ensure the diversity of the population and the convergence speed of the population.

\subsection{Network structure of SOM}

In GWO-SFMEA, the structure of SOM for the bi-objective and tri-objective problems is set to be one-dimensional $(1 \times 100)$ and two-dimensional $(7 \times 15)$ respectively. In order to study the influence of the SOM structure on the performance metrics $I G D$ and $H V$ of the algorithm, the SOM structure was changed to be two-dimensional $(10 \times 10) \mathrm{SOM}$ in bi-objective problem and one-dimensional $(1 \times 105)$ in triobjective problem. The two kinds of SOM structures were both run on GLT1-GLT6 for 30 times. The IGD and $H V$ values obtained from each instance are compared with the unmodified ones. The results are shown in Fig. 11.

It can be observed from Fig 11(a) that for the bi-objective instances, the $I G D$ value on GLT1-2 is smaller under the one-dimensional SOM structure. However, the $I G D$ value of GLT3-4 is smaller under the two-dimensional structure. For the tri-objective problem, the $I G D$ value is smaller under the two-dimensional structure.

It can be seen from (b) that the change of the SOM structure has little effect on the $H V$ value on all GLT problems. Strictly speaking, the $H V$ value on the bi-objective problem is slightly larger under the one-dimensional structure, and that on the tri-objective problem is a little larger under the two-dimensional structure.

In conclusion, the bi-objective problem has no obvious preference for one-dimensional or two-dimensional SOM, but the tri-objective instance prefers two-dimensional SOM structure.

\section{Conclusions}

In this paper, a new MOEA called grey wolf optimization based self-organizing fuzzy multi-objective evolution algorithm was proposed. In algorithms which combine SOM with MOEAs, the initial weight of self-organizing map is generated randomly. However, the result depends heavily on the initial weight. Therefore, this paper optimized the initial weights of SOM through grey wolf optimization algorithm. At the same time, SOM can be used to map high-

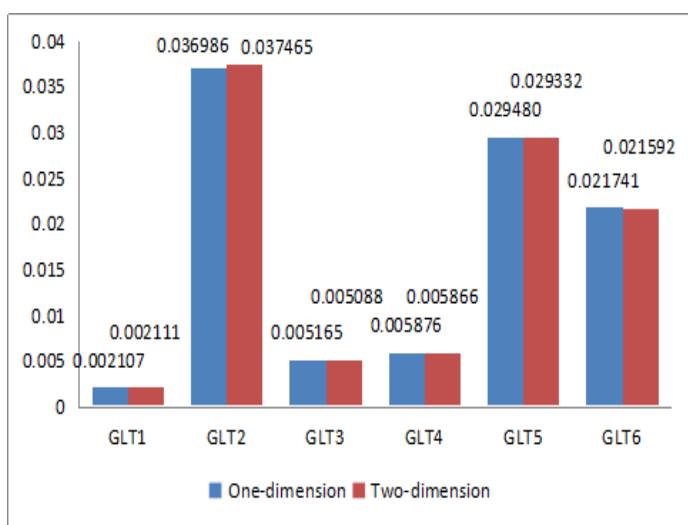

(a) The Influence of SOM Structure on IGD

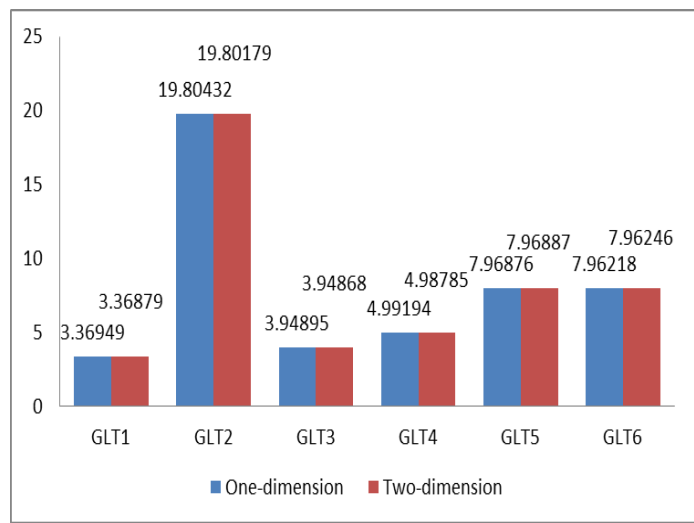

(b) The Influence of SOM Structure on $H V$

Fig. 11: The Influence of SOM Structure on $I G D$ and $H V$.

dimensional information to low-dimensional space while maintaining feature distribution invariance and building neighborhood relationships between individuals. In addition, the suboptimal solutions produced in the early optimization process in most MOEAs induce the algorithm to converge prematurely with great probability. To avoid this problem, this paper first utilized FDE operator to generate a new initial solution. Then the PM operator was used to mutate the new solution and boundary processing was performed. FDE dynamically adjusts the weighting parameter $F$ in the difference operator by constructing a fuzzy inference system, which can ensure high-quality new solutions during the generation of offspring and effectively improve the convergence and diversity of the algorithm.

In order to verify the performance of the proposed algorithm, GWO-SFMEA and other state-of-the-art algorithms were test on the GLT and WFG test functions with complex PF shapes and/or complex PS shapes, respectively. Through experiments, it can be found that the proposed algorithm is significantly better than other algorithms at the $95 \%$ confidence level. Through analysis of the sensitivity of GWOSFMEA to the SOM structure, it can be seen that the SOM structure has no obvious preference for the bi-objective prob- 
lem, but the tri-objective problem prefers the two-dimensional SOM structure. In addition, the combination of fuzzy systems with EAs can greatly improve the convergence and diversity of evolutionary algorithms.

Acknowledgements This work was supported in part by the National Natural Science Foundation of China (No. 11371130, 12071179), Soft science research program of Fujian Province (No. B19085), the project of Education Department of Fujian Province (No. JT180263), the Youth Innovation Fund of Xiamen City (3502Z20206020), the open fund of Key Laboratory of Applied Mathematics of Fujian Province University (Putian University) (No. SX201906) and Digital Fujian big data modeling and intelligent computing institute, Pre-Research Fund of Jimei University.

\section{Compliance with ethical standards}

Conflict of interest All authors declare that they have no conflict of interest.

Ethical approval This article does not contain any studies with human participants or animals performed by any of the authors.

Informed consent Informed consent was obtained from all individual participants included in the study.

Authorship contributions Jialiang Xie gave the overall framework of the paper and introduction, and Shanli Zhang completed the experiment and the first draft. Honghui Wang modified the grammar of the paper, and Dongrui Wu coordinated the paper.

\section{References}

Bošković B, Brest J (2018) Protein folding optimization using differential evolution extended with local search and component reinitialization. Information Sciences 454455:178-199

Cai X, Xiao Y, Li M, Hu H, Li X (2021) A grid-based inverted generational distance for multi/many-objective optimization. IEEE Transactions on Evolutionary Computation 25(99):21-34

Chen D, Zou F, Lu R, Wang X (2018) A hybrid fuzzy inference prediction strategy for dynamic multi-objective optimization. Swarm \& Evolutionary Computation 43:147165

Durillo JJ, Nebro AJ, Luna F, Alba E (2008) Solving threeobjective optimization problems using a new hybrid cellular genetic algorithm. In: Proceedings of the 10th international conference on Parallel Problem Solving from Nature: PPSN X, pp 661-670

Elhariri E, El-Bendary N, Hassanien AE (2016a) A hybrid classification model for EMG signals using grey wolf optimizer and SVMs 407:297-307
Elhariri E, El-Bendary N, Hassanien AE, Abraham A (2016b) Grey wolf optimization for one-against-one multi-class support vector machines. In: Soft Computing \& Pattern Recognition, pp 7-12

Gu F, lin Liu H, Tan KC (2012) A multiobjective evolutionary algorithm using dynamic weight design method. International Journal of Innovative Computing Information \& Control Ijicic 8(5B):3677-3688

Huband S, Barone LC, While L, Hingston PF (2005) A scalable multi-objective test problem toolkit. Lecture Notes in Computer Science 3410:280-295

Jing L, Qianqian G, Caitong Y, Boyang Q (2019) Selforganizing multi-objective particle swarm optimization algorithm. Application Research of Computers 36(8):2311-2316(2348)

Kohonen, Teuvo (1990) The self-organizing map. IEEE Proc Icnn 78(9):1464-1480

Korashy A, Kamel S, Nasrat L, Jurado F (2020) Developed multi-objective grey wolf optimizer with fuzzy logic decision-making tool for direction overcurrent relays coordination. Soft Computing 24(17):13305-13317

Lara A, Sanchez G, Coello Coello C, Schutze O (2010) HCS: A new local search strategy for memetic multiobjective evolutionary algorithms. IEEE Transactions on Evolutionary Computation 14(1):112-132

Li H, Zhang Q (2009) Multiobjective optimization problems with complicated pareto sets, MOEA/D and NSGAII. IEEE Transactions on Evolutionary Computation 13(2):284-302

Marler RT, Arora JS (2004) Survey of multi-objective optimization methods for engineering. Structural \& Multidisciplinary Optimization 26(6):369-395

Melin P, Olivas F, Castillo O, Valdez F, Soria J, Valdez M (2013) Optimal design of fuzzy classification systems using PSO with dynamic parameter adaptation through fuzzy logic. Expert Systems with Applications 40(8):3196-3206

Mendes R, Mohais AS (2005) DynDE: a differential evolution for dynamic optimization problems. In: IEEE Congress on Evolutionary Computation, pp 2808-2815

Ming M, Wang R, Zha Y, Zhang T (2017) Pareto adaptive penalty-based boundary intersection method for multiobjective optimization. Information Sciences 414:158174

Mirjalili S, Mirjalili SM, Lewis A (2014) Grey wolf optimizer. Advances in Engineering Software 69(3):46-61

Mustaffa Z, Sulaiman MH, Kahar MNM (2015) Training LSSVM with GWO for price forecasting. In: 2015 International Conference on Informatics, Electronics and Vision (ICIEV), pp 1-6

Nebro AJ, Durillo JJ, Luna F, Dorronsoro B, Alba E (2009) MOCell: A cellular genetic algorithm for multiobjective optimization. International Journal of Intelligent Systems 
24(7):726-746

Norouzi K, Rakhshandehroo GR (2011) A self organizing map based hybrid multi-objective optimization of water distribution networks. Iranian Journal of Science and Technology Transaction B Engineering 35(1):105-119

Olivas F, Valdez F, Castillo O, Gonzalez CI, Martinez G, Melin P (2017) Ant colony optimization with dynamic parameter adaptation based on interval type-2 fuzzy logic systems. Applied Soft Computing 53:74-87

Price KV, Storn RM, Lampinen JA (2005) Differential Evolution-A Practical Approach to Global Optimization. Springer-Verlag, Berlin

Sankhwar S, Gupta D, Ramya KC, Rani SS, Lakshmanaprabu SK (2019) Improved grey wolf optimizationbased feature subset selection with fuzzy neural classifier for financial crisis prediction. Soft Computing 24(4):101110

Santiago A, Dorronsoro B, Nebro AJ, Durillo JJ, Fraire HJ (2019) A novel multi-objective evolutionary algorith$m$ with fuzzy logic based adaptive selection of operators:FAME. Information Sciences 471:233-251

Saremi S, Mirjalili SZ, Mirjalili SM (2015) Evolutionary population dynamics and grey wolf optimizer. Neural Computing \& Applications 26(5):1257-1263

Shen Y, Ge G, Jia L, Meng B, Wang Y (2019) Multiobjective particle swarm optimization based on fuzzy optimality. IEEE Access 7(99):101513-101526

Sivanandam N, Sai S, Deepa SN (2007) Introduction to fuzzy logic using MATLAB. Springer, Berlin

Song H, Miao C, Shen Z (2007) Fuzzy cognitive map learning based on multi-objectiveparticle swarm optimization. In: Proceedings of the 9th annual conference on Genetic and evolutionary computation, pp 339-339

Storn R (1947) On the usage of differential evolution for function optimization. In: Fuzzy Information Processing Society, Nafips Biennial Conference of the North American, pp 519-523

Teuvo, Kohonen (1998) The self-organizing map. Neurocomputing 21(1):1-6

Wang L, Zhang Q (2016) Constrained subproblems in decomposition based multiobjective evolutionary algorithm. IEEE Transactions on Evolutionary Computation 20(3):475-480

Yogesh G, Ashish S (2018) A new swarm-based efficient data clustering approach using KHM and fuzzy logic. Soft Computing 23:145-162

Zhang H, Song S, Zhou A, Gao XZ (2015) A multiobjective cellular genetic algorithm based on 3D structure and cosine crowding measurement. International Journal of Machine Learning \& Cybernetics 6(3):487-500

Zhang H, Zhou A, Song S, Zhang Q, Zhang J (2016) A selforganizing multiobjective evolutionary algorithm. IEEE Transactions on Evolutionary Computation 20(5):792-
806

Zhang Q, Zhou A, Jin Y (2008) RM-MEDA: A regularity model-based multiobjective estimation of distribution algorithm. IEEE Transactions on Evolutionary Computation 12(1):41-63

Zhou A, Zhang Q (2016) Are all the subproblems equally important? Resource allocation in decomposition-based multiobjective evolutionary algorithms. IEEE Transactions on Evolutionary Computation 20(1):52-64

Zhou A, Zhang Q, Jin Y, Tsang E (2005) A model-based evolutionary algorithm for bi-objective optimization. In: IEEE Congress on Evolutionary Computation, vol 3, pp 2568-2575

Zhou A, Zhang Q, Jin Y (2009) Approximating the set of pareto-optimal solutions in both the decision and objective spaces by an estimation of distribution algorithm. IEEE Transactions on Evolutionary Computation 13(5):1167-1189

Zitzler E, Thiele L (1999) Multiobjective evolutionary algorithms: a comparative case study and the strength pareto approach. IEEE Transactions on Evolutionary Computation 3(4):257-271

Zou F, Chen D, Xu Q, Lu R (2020) A new prediction strategy combining T-S fuzzy nonlinear regression prediction and multi-step prediction for dynamic multiobjective optimization. Swarm \& Evolutionary Computation 59:100749-100768 


\section{Figures}

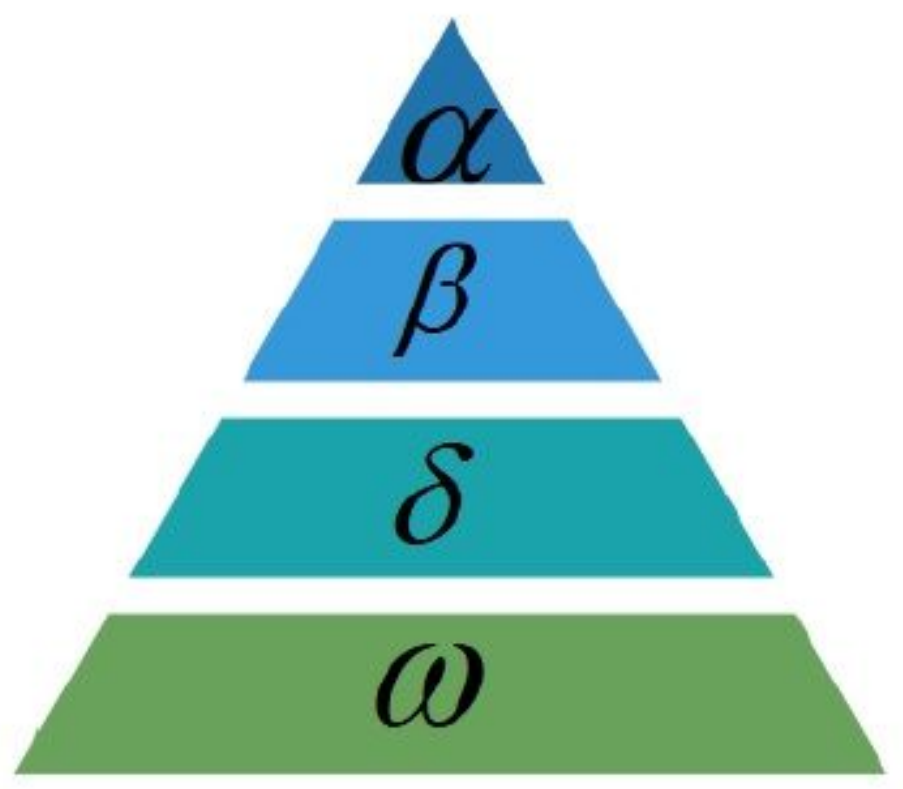

Figure 1

Hierarchy of grey wolves

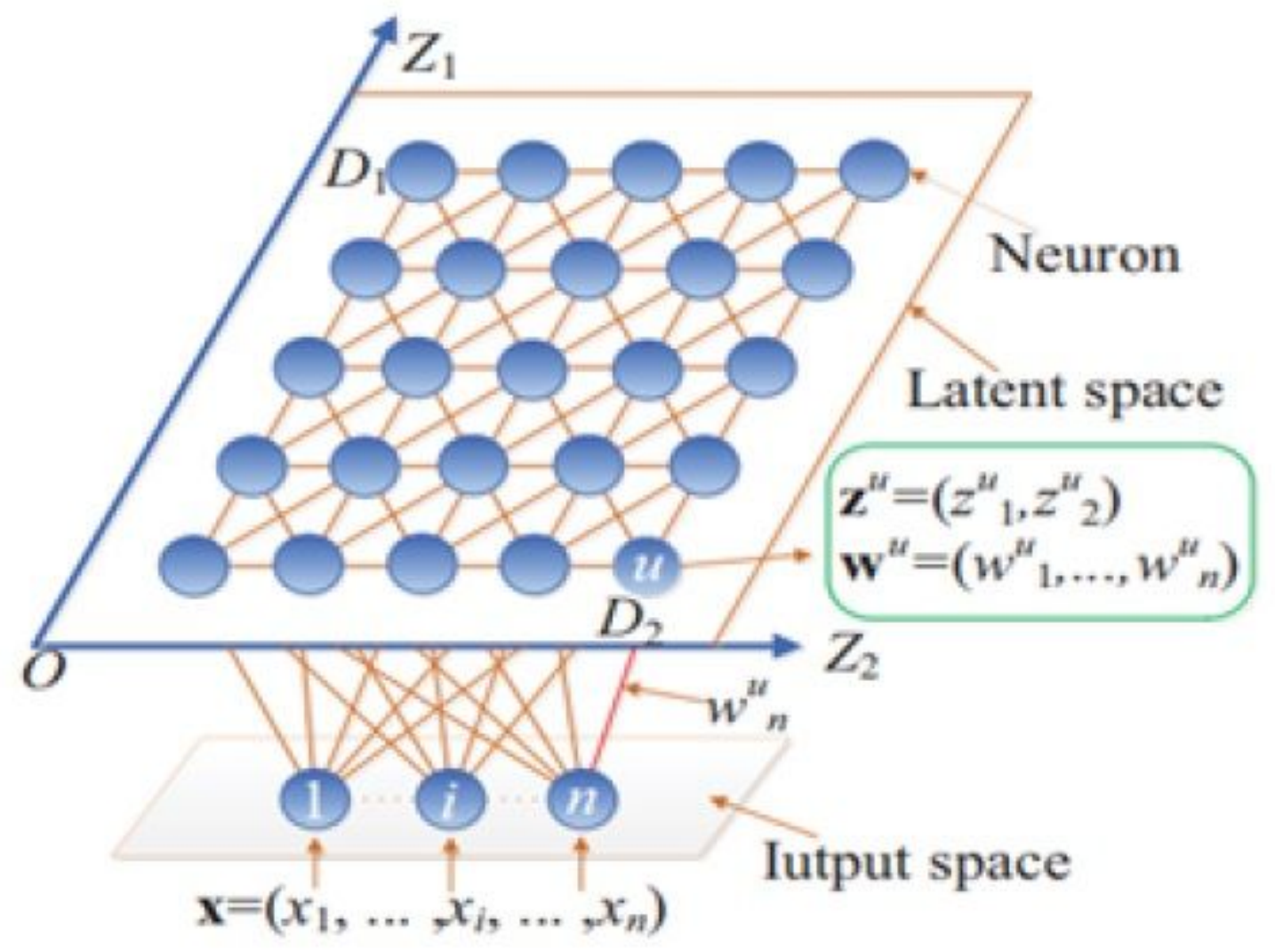

Figure 2 
An illustration of a 2-dimensional SOM

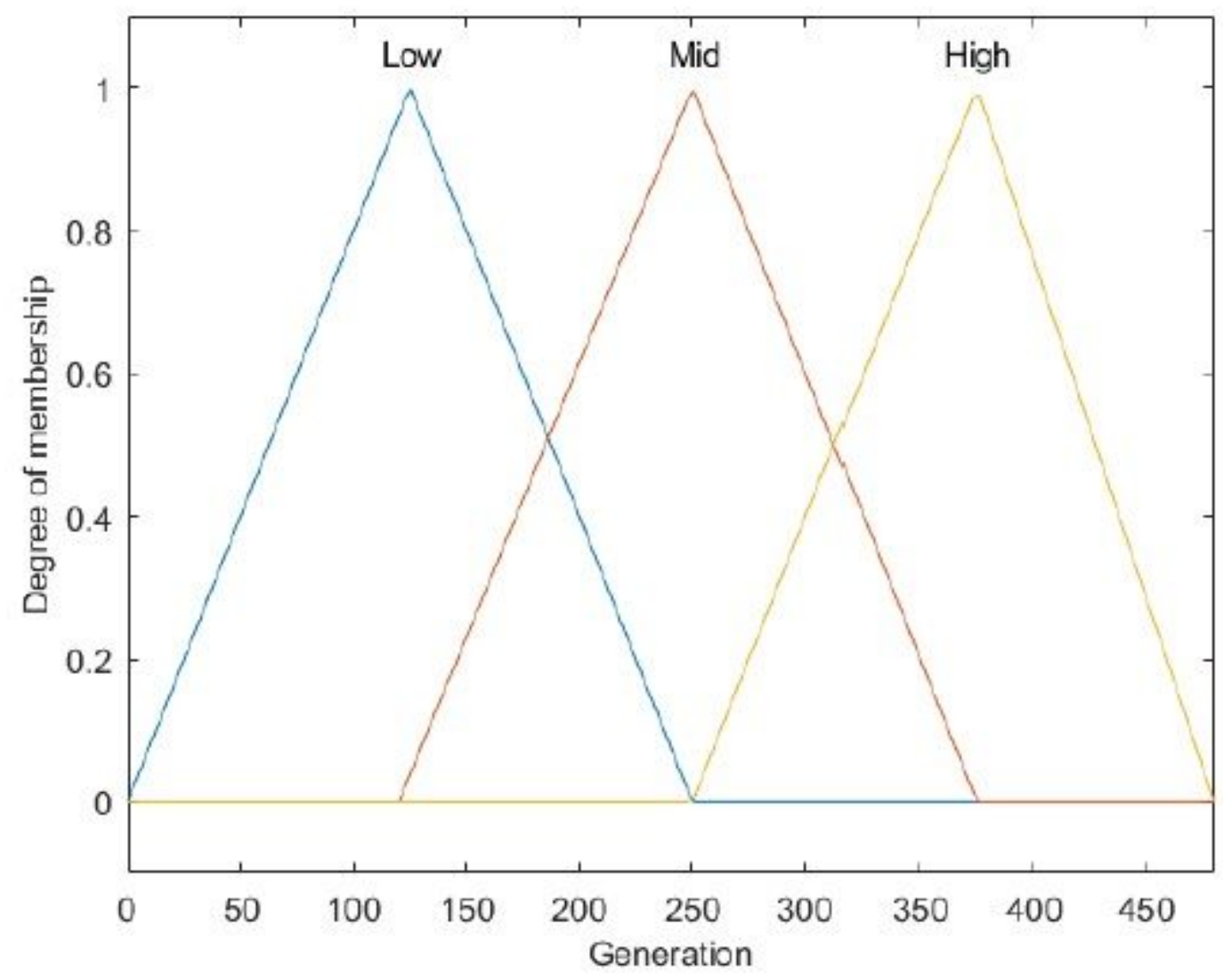

Figure 3

Generation membership function and the representation of linguistic terms 




Figure 4

Utilization membership function and the representation of linguistic terms 


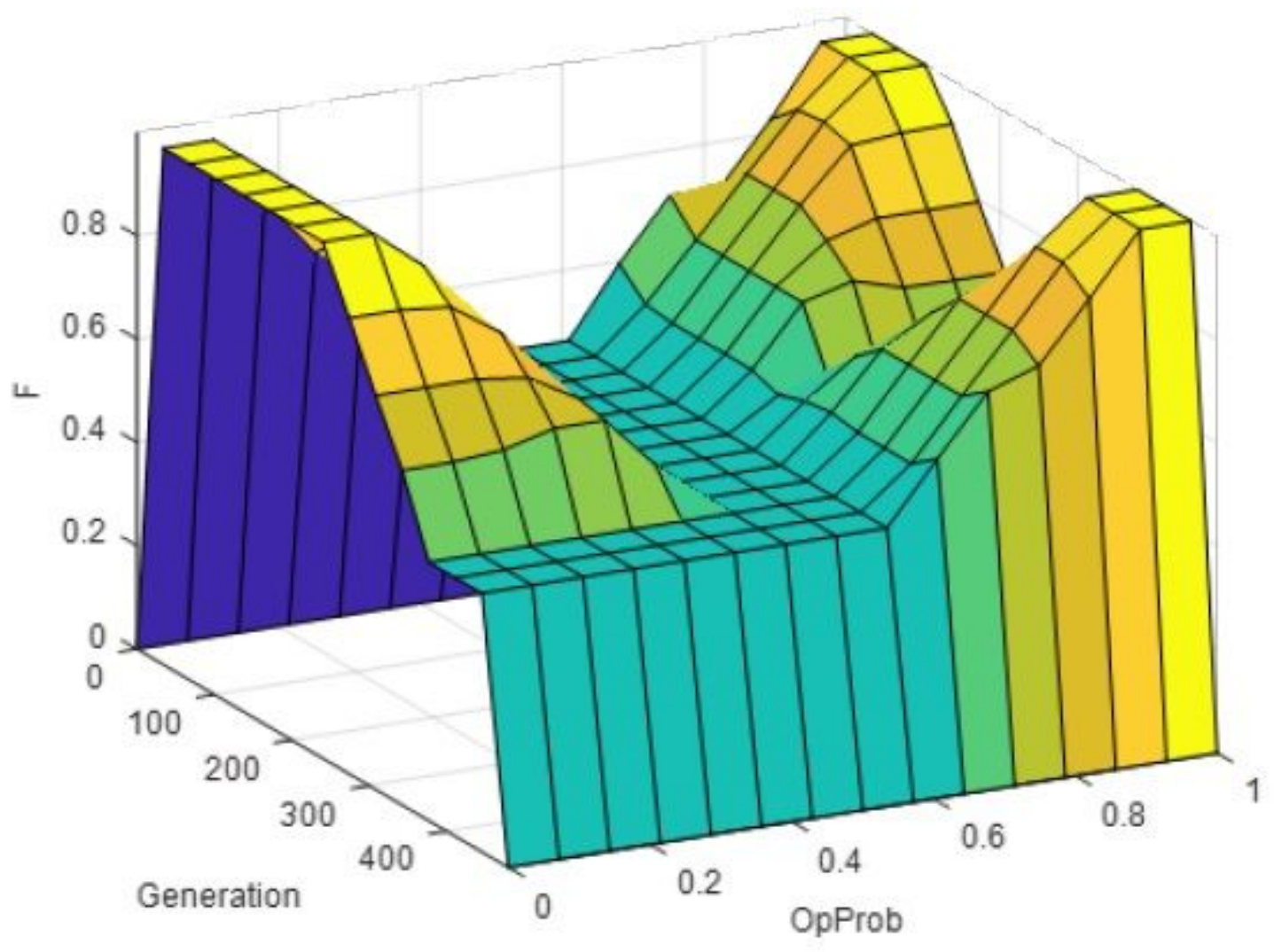

Figure 5

Surface function graph of fuzzy inference system 


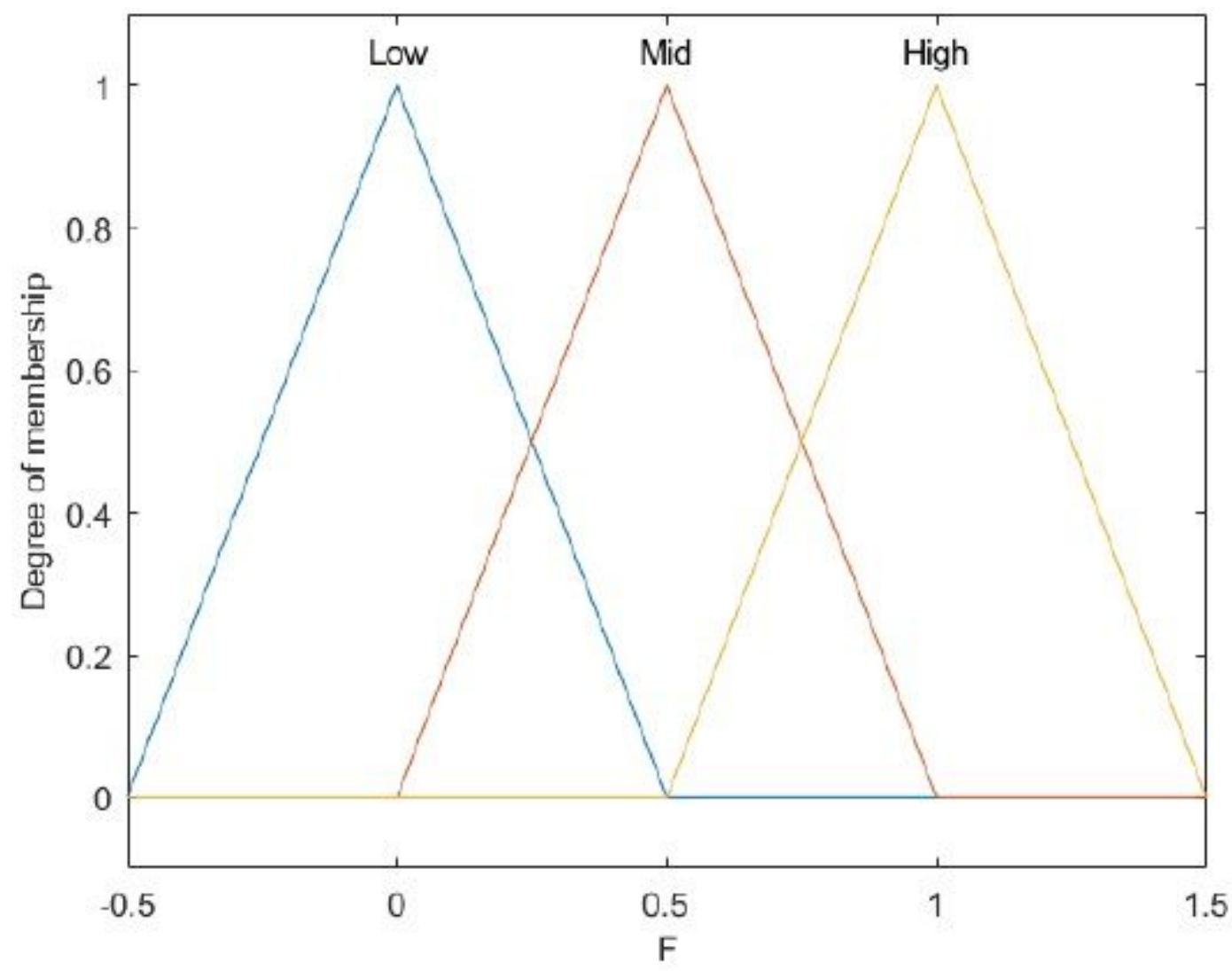

Figure 6

The membership function and linguistic terms of output $F$ 


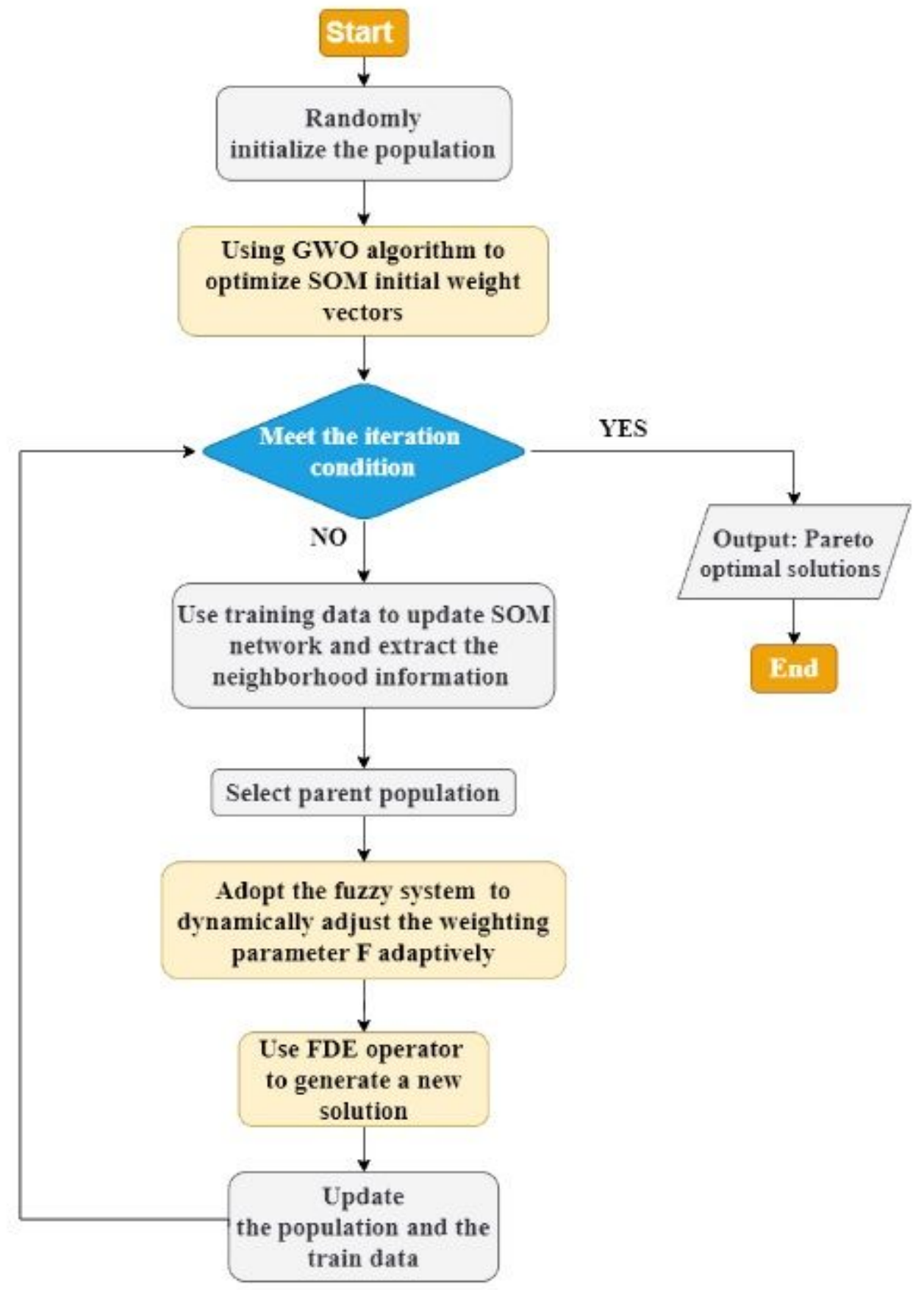

Figure 7

The GWO-SFMEA framework. 

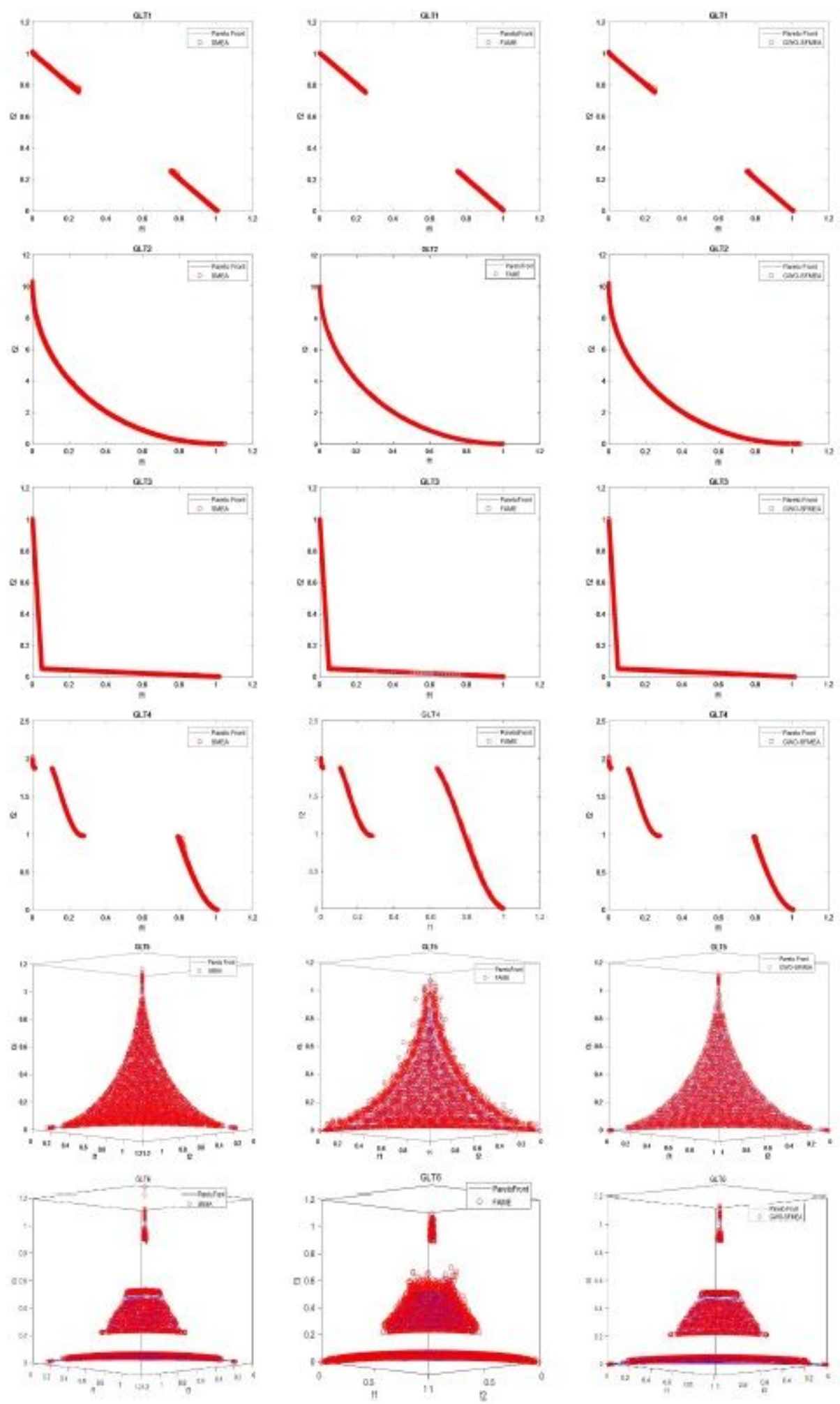

Figure 8

The final populations in the objective space obtained by SMEA, FAME and GWO-SFMEA on GLT1-GLT6 over 30 runs. 


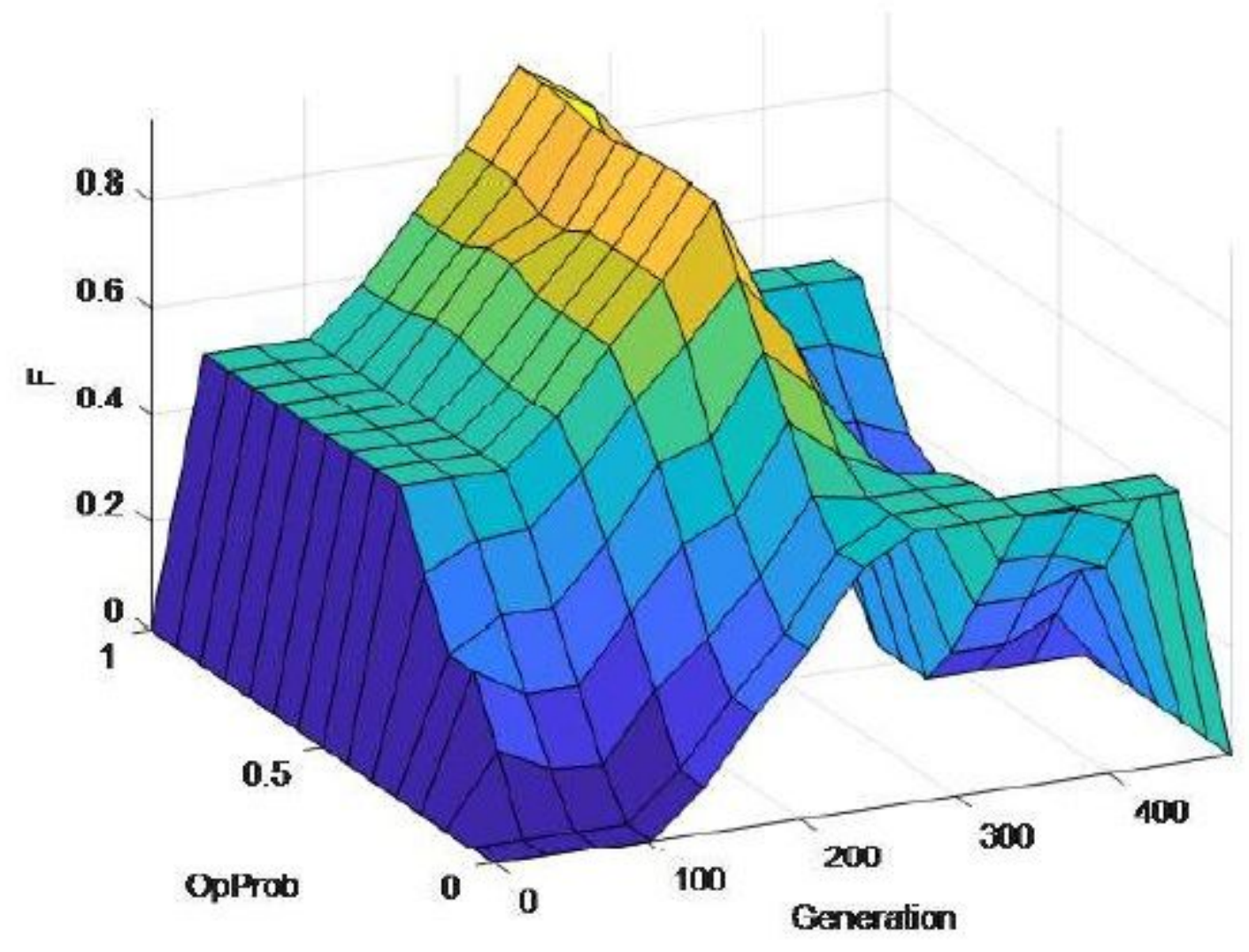

Figure 9

Output surface of fuzzy system with weighted parameter F. 


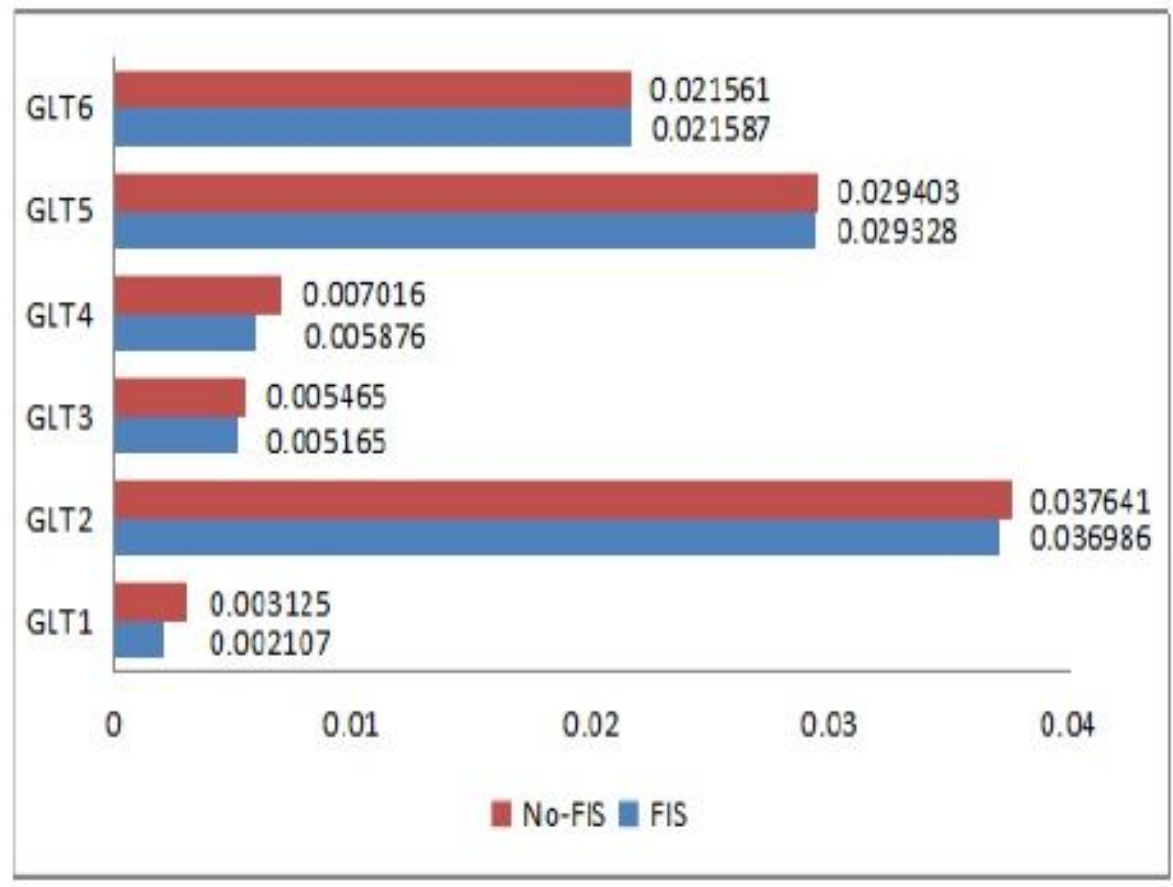

(a) The impact of fuzzy system on $I G D$

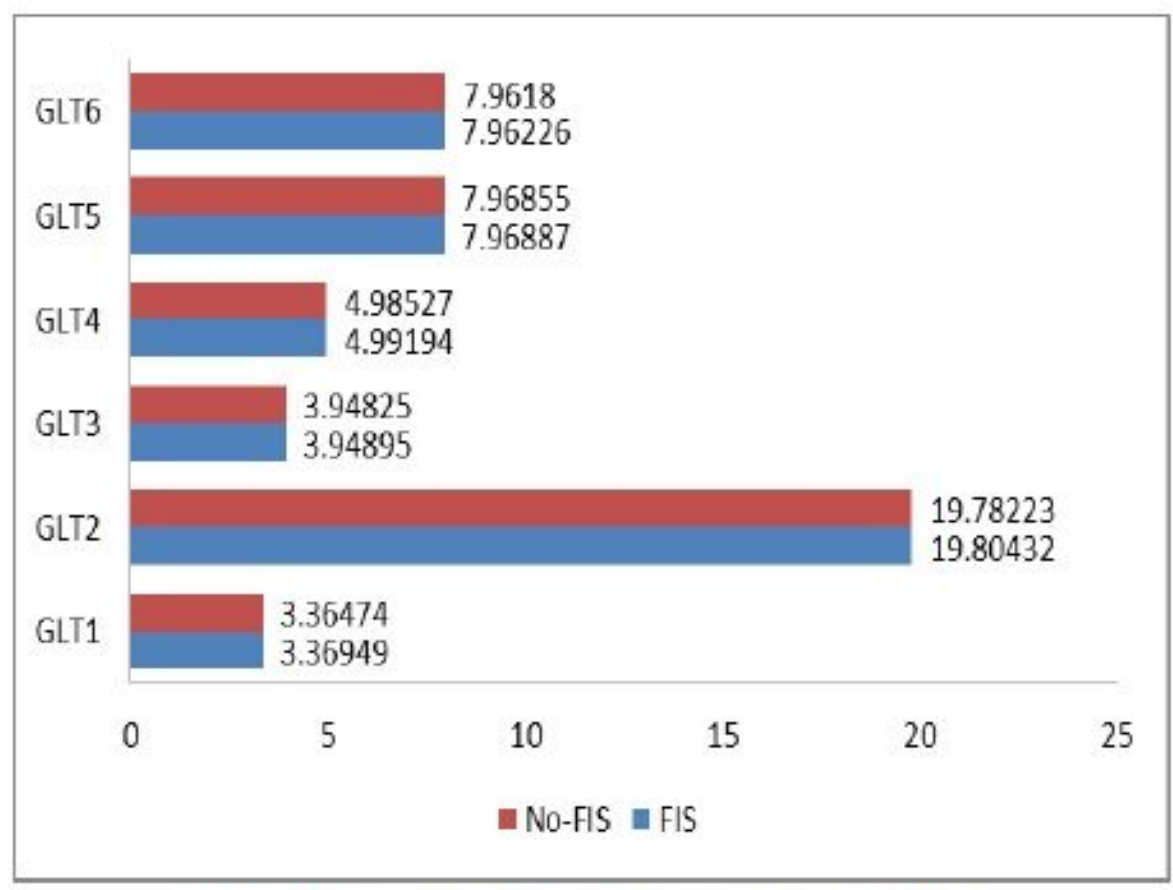

(b) The impact of fuzzy system on $H V$

Figure 10

The impact of fuzzy system on IGD and HV. 


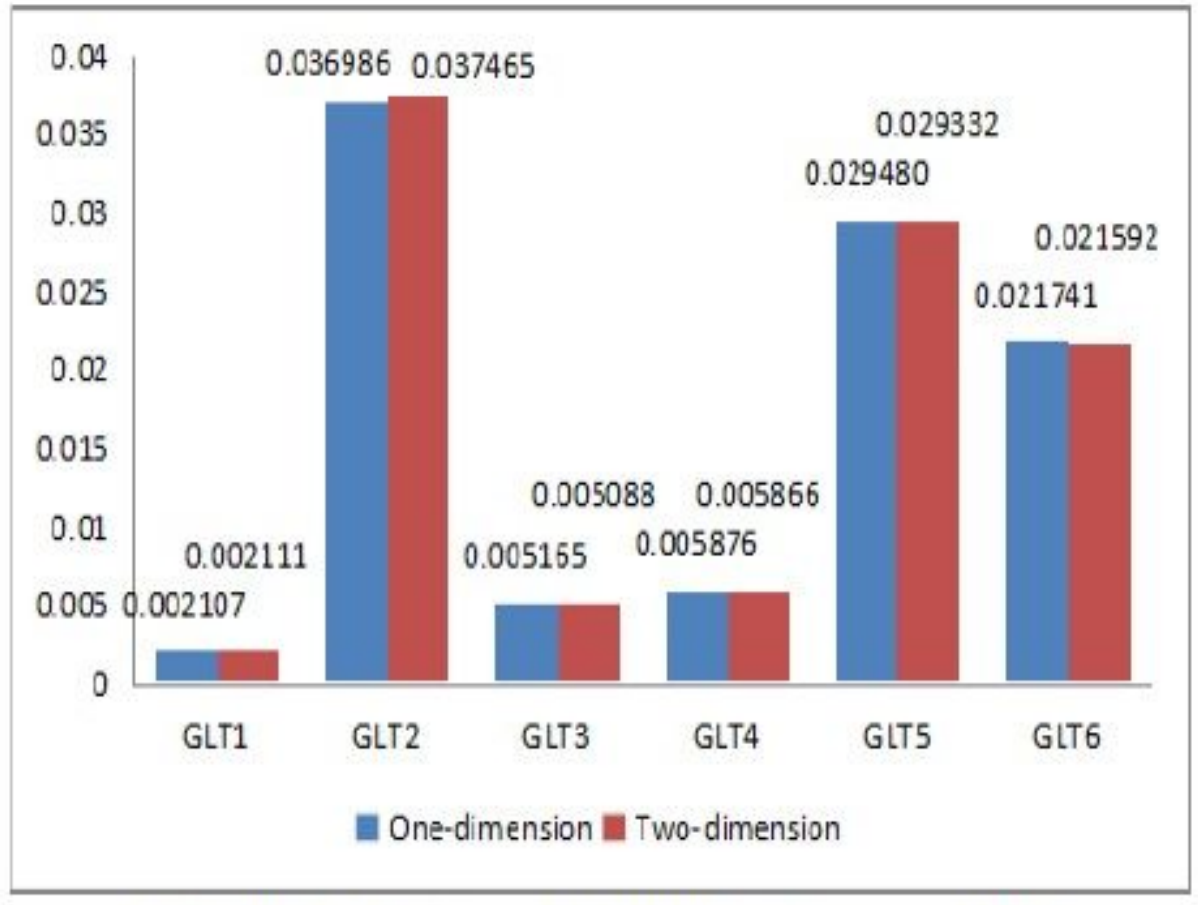

(a) The Influence of SOM Structure on IGD

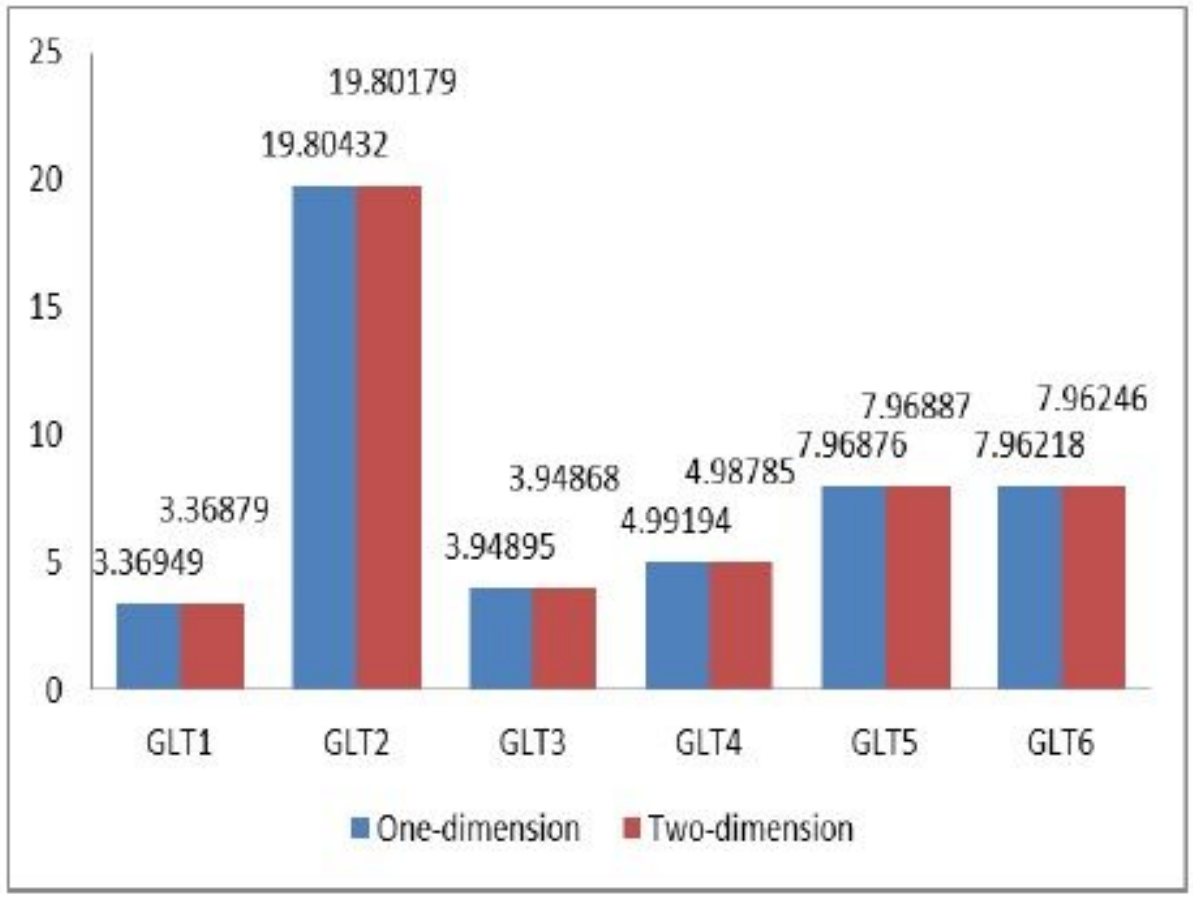

(b) The Influence of SOM Structure on $H V$

Figure 11

The Influence of SOM Structure on IGD and HV. 